OPEN ACCESS

Edited by:

Biqin Dong,

Fudan University, China

Reviewed by:

Junhui Shi,

Zhejiang Lab, China

Chengbo Liu,

Chinese Academy of Sciences (CAS),

China

*Correspondence:

Silviu-Vasile Bodea

silviu.bodea@helmholtz-muenchen.de

Gil Gregor Westmeyer

gil.westmeyer@tum.de

Specialty section:

This article was submitted to

Brain Imaging Methods,

a section of the journal

Frontiers in Neuroscience

Received: 18 January 2021

Accepted: 08 March 2021

Published: 10 June 2021

Citation:

Bodea S-V and Westmeyer GG (2021) Photoacoustic Neuroimaging -

Perspectives on a Maturing Imaging

Technique and its Applications

in Neuroscience.

Front. Neurosci. 15:655247.

doi: 10.3389/fnins.2021.655247

\section{Photoacoustic Neuroimaging - Perspectives on a Maturing Imaging Technique and its Applications in Neuroscience}

\author{
Silviu-Vasile Bodea ${ }^{1,2 *}$ and Gil Gregor Westmeyer ${ }^{1,2 *}$ \\ 'Department of Chemistry and School of Medicine, Technical University of Munich (TUM), Munich, Germany, ${ }^{2}$ Institute \\ for Synthetic Biomedicine, Helmholtz Center Munich, Munich, Germany
}

A prominent goal of neuroscience is to improve our understanding of how brain structure and activity interact to produce perception, emotion, behavior, and cognition. The brain's network activity is inherently organized in distinct spatiotemporal patterns that span scales from nanometer-sized synapses to meter-long nerve fibers and millisecond intervals between electrical signals to decades of memory storage. There is currently no single imaging method that alone can provide all the relevant information, but intelligent combinations of complementary techniques can be effective. Here, we thus present the latest advances in biomedical and biological engineering on photoacoustic neuroimaging in the context of complementary imaging techniques. A particular focus is placed on recent advances in whole-brain photoacoustic imaging in rodent models and its influential role in bridging the gap between fluorescence microscopy and more noninvasive techniques such as magnetic resonance imaging (MRI). We consider current strategies to address persistent challenges, particularly in developing molecular contrast agents, and conclude with an overview of potential future directions for photoacoustic neuroimaging to provide deeper insights into healthy and pathological brain processes.

Keywords: optoacoustic imaging, functional brain imaging, molecular contrast agents, calcium and voltage sensors, stroke, brain tumors, image-guided therapy, translational photoacoustic imaging

\section{INTRODUCTION}

Understanding the mechanisms by which the brain gives rise to inner experience, cognition, and behavior remains a central objective of fundamental neuroscience, which will also strongly foster advances in causal therapies of neuropsychiatric diseases.

Mapping spatiotemporal patterns across the vastly different granularities of the brain becomes increasingly important for testing specific circuit models and monitoring pathological processes and therapeutic interventions (Friston, 1994; Sporns et al., 2005; Sporns, 2013).

As a still younger imaging technique, photoacoustic (or synonymously optoacoustic) imaging is entering a crowded field of specialized anatomical and functional imaging modalities. There have been a series of excellent reviews of photoacoustic imaging (PAI) technology which include in-depth comparisons with optical and non-optical imaging techniques in terms of sensitivity to 
contrast agents, penetration depth, and resolution (Ntziachristos, 2010; Devor et al., 2012; Hu and Wang, 2013; DeánBen et al., 2017; Ovsepian et al., 2017; Jeon et al., 2019; Hosseinaee et al., 2020).

This review focuses mainly on photoacoustics' current capabilities for preclinical brain imaging to extract physiological and molecular contrast and on how the modality can synergize with complementary neuroimaging techniques. We will mostly discuss preclinical research in mouse models used in most current PA neuroimaging studies because the mouse remains the most important organism for modeling human disease, and the dimensions of the mouse brain match well with PA capabilities in terms of tissue penetration and resolution (McGraw et al., 2017). We will then point out emerging opportunities for translation of PA neuroimaging into the clinic.

In structural neuroimaging, electron microscopy (EM) and light-sheet microscopy combined with tissue clearing are systematically improving our maps of brain connections, the so-called connectome (Sporns et al., 2005). Multiphoton microscopy is limited in penetration depth, while computed tomography (CT), magnetic resonance imaging (MRI), and positron emission tomography (PET) offer non-invasive structural characterization of entire brains albeit at lower spatial resolution (Ntziachristos and Razansky, 2010).

In vivo, functional and molecular neuroimaging at microscopic scales is dominated by multiphoton techniques, while implantable light guides and miniaturized microscopes illuminate network dynamics in freely moving animals (Malvaut et al., 2020). Widefield epifluorescence, coupled with intrinsic optical imaging (IOS), can cover the entire cortex of small rodents but only superficial layers. Functional MRI (fMRI), functional ultrasound (fUS), and PET can image dynamic processes across the entire mammalian brain at lower spatial and temporal resolutions. For this reason, MRI and PET are probably still the most influential neuroimaging tools, which also connect to neuroscientific studies in humans.

\section{IMAGING BASED ON THE PHOTOACOUSTIC EFFECT}

The photoacoustic effect relies on the differential thermoelastic expansion of materials after irradiation with non-ionizing electromagnetic waves, causing wideband sound emission (Bell, 1880). Theoretical work into the detection of laser-induced stress waves (Oraevsky et al., 1993, 1997) paved the way for laserbased photoacoustic microscopes (Wada et al., 1986; Cross et al., 1987; Masujima et al., 1988; Maslov et al., 2005) as well as tomographic imaging setups optimized for imaging biological tissue (Kruger et al., 1995; Esenaliev et al., 1996; Oraevsky et al., 1996; Wang et al., 2003b) as detailed in a recent review (Manohar and Razansky, 2016).

Current biomedical PA imaging systems routinely use tunable laser systems in the visible and infrared spectrum. The laser pulses are partially absorbed by tissue chromophores, leading to a conversion into heat with a local temperature increase on the order of millikelvins (Yao and Wang, 2014). The resulting thermoelastic expansion generates a transient pressure increase that propagates through the sample as a broadband acoustic wave which can be recorded by ultrasound detectors and computationally reconstructed to map the distribution of photoabsorbers in the tissue (Ntziachristos, 2010).

The main driver for image contrast is, therefore, differential laser light absorption in biological media. Multispectral laser excitation consequently can localize specific endogenous or exogenous chromophores based on their specific photoacoustic spectra (Razansky et al., 2011).

Because acoustic scattering is orders of magnitude weaker than optical scattering, PA imaging can reach a higher spatial resolution and tissue penetration than traditional, purely optical techniques but still access the rich information contained in endogenous and exogenous photoabsorbing molecules (Ntziachristos and Razansky, 2010).

\section{DETECTION GEOMETRIES FOR PHOTOACOUSTIC NEUROIMAGING}

Over the last years, photoacoustic technology has given rise to specialized imaging geometries that find different tradeoffs in spatial and temporal resolution and penetration depth (DeánBen et al., 2017). We will briefly summarize the main categories to illustrate practical aspects regarding which samples can be accessed and which results may be expected (Figure 1).

PA microscopy (PAM) can be realized either by restricting the photoacoustic signal generation by focusing a laser beam, so-called optical resolution (OR), or by illuminating the sample more broadly but detecting the ultrasound only from a focal volume, termed acoustic resolution (AR).

OR-PAM relies on tight laser beam focusing onto a selective spot in the tissue and receiving the emitted ultrasound from that optical focal cone on a transducer immersed in a coupling medium. Resolution is limited to the smallest achievable optical focal spot size, and imaging depth is limited to an optical transport mean free path $(\sim 1 \mathrm{~mm})$ (Maslov et al., 2008; Hu et al., 2011b; Hu and Wang, 2013; Yao and Wang, 2013). Thus, similar to optical microscopes, the spatial resolution is diffractionlimited (Deán-Ben et al., 2017; Seeger et al., 2020). OR-PAM necessitates raster scanning across the sample and is often implemented in hybrid microscopes with confocal, multiphoton, or light-sheet microscopy to complement the photoabsorbance maps (Seeger et al., 2020). Since acoustic coupling of the sample with the transducer can be impractical, PA microscopes have been developed which do not require physical coupling between the microscope and sample tissue. Remote sensing photoacoustic microscopy (PARS) is designed to resolve this by co-focusing a continuous probe beam together with the nanosecond excitation beam. The pressure changes from the absorbed light can be detected as changes in the intensity of the reflected probe beam (Bell et al., 2017; Hajireza et al., 2017). This setup may achieve contract-free diffraction-limited resolution at distances of up to $2.5 \mathrm{~cm}$ to the imaged tissue (Hajireza et al., 2017) making it suitable for intraoperative applications (Abbasi et al., 2019; Ecclestone et al., 2020). 

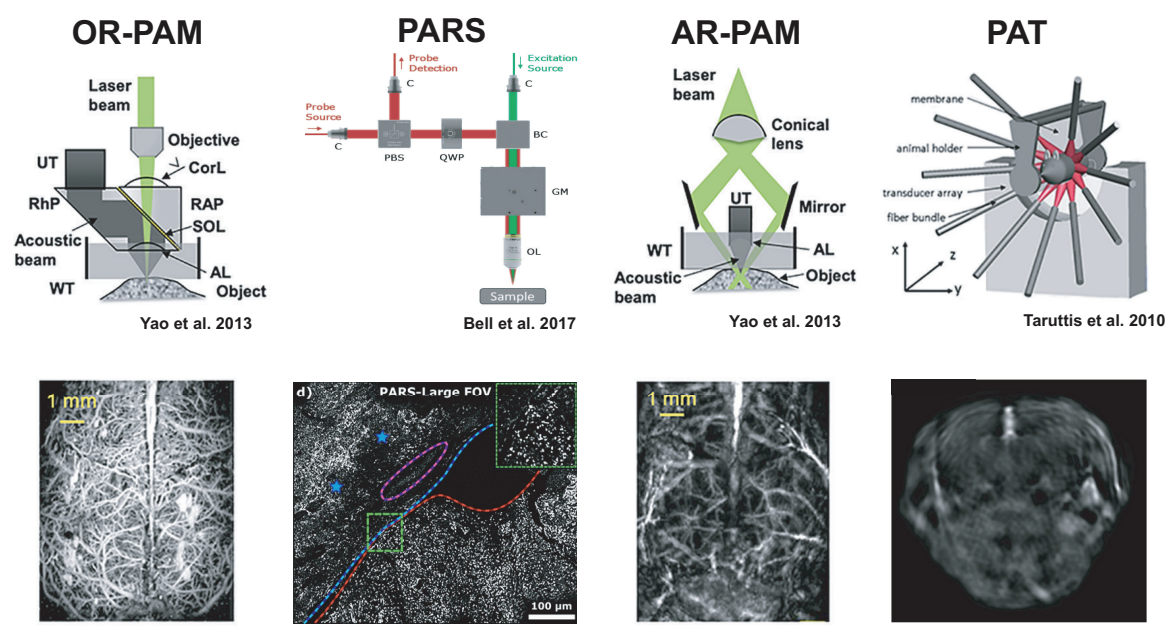

Ecclestone et al 2020

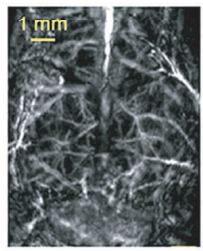

Yao et al. 2013

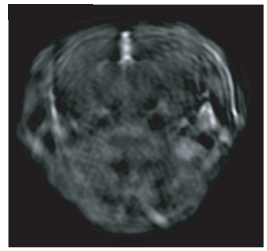

Burton et al.2013
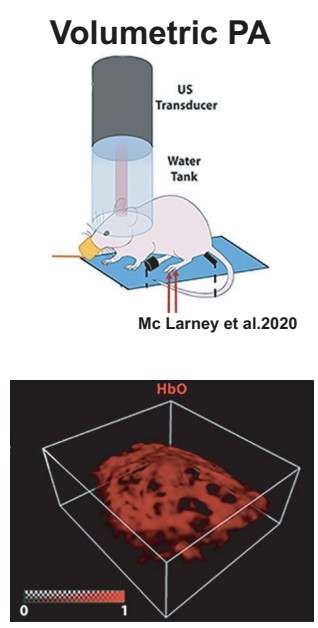

Mc Larney et al.2020

FIGURE 1 | Detection geometries for photoacoustic imaging with example results from neuroimaging. The figures were adapted from the respective references with permission from the authors.

AR-PAM, in distinction, works with laser excitation that can cover the entire acoustic detection area because the lateral resolution is defined by the acoustic focusing capabilities ( $\mathrm{Li}$ et al., 2006; Zhang et al., 2006; Park et al., 2014). Image formation occurs from raster scanning of focused transducers and stacking depth profiles from individual laser shots. ARPAM implementations can reach imaging depths of $5 \mathrm{~mm}$ while maintaining $7 \mu \mathrm{m}$ axial and $30 \mu \mathrm{m}$ lateral resolutions (Omar et al., 2014). One such implementation, raster-scan optoacoustic mesoscopy (RSOM), can be productively combined with ORPAM, multiphoton excitation fluorescence, second, and third harmonic generation microscopy (Soliman et al., 2015).

Rather than scanning a single transducer across the specimen, photoacoustic tomography (PAT) uses a multi-element ultrasound detector array to enable the parallel acquisition of an imaging plane through the specimen with a single-shot laser excitation (Kruger et al., 1995; Hoelen et al., 1998; Buehler et al., 2010; Razansky et al., 2011). Light is typically delivered through optical fiber bundles from the side of the transducer array, and tomographic reconstruction of the photoacoustic signals is used to compute the $2 \mathrm{D}$ images (Buehler et al., 2010; Lutzweiler and Razansky, 2013). Multiple slices can be acquired by translating either the specimen or the transducer array. By using rapidly tunable pulsed-lasers in visible or nearinfrared wavelengths, photoacoustic spectra can be acquired at each subvolume (MSOT), which can allow mapping of the distribution of different photoabsorbers based on characteristic spectra via spectral unmixing methods (Taruttis et al., 2010; Razansky et al., 2011; Tzoumas et al., 2014).

By arranging a two-dimensional array of ultrasonic detectors on a spherical surface, entire image volumes can be reconstructed from single laser pulses without a scanning procedure (DeánBen and Razansky, 2013; Xiang et al., 2013; Deán-Ben et al., 2015a). This volumetric PA imaging can also be augmented with multi-spectral information via tunable lasers (Luís Deán-Ben and Razansky, 2014). For practical reasons, laser illumination is usually performed through a central opening of the spherical transducer array, and fluorescence imaging can be added by using coherent fiber bundles (Chen et al., 2017). Current volume rates are limited by the pulse repetition frequency of available laser systems $\sim 100 \mathrm{~Hz}$ but could exceed those of ultrasound. Because of the large volume rates, this scanless image acquisition is very attractive for whole-brain neuroimaging in rodents and other animal models.

\section{NEUROANATOMY OF ANIMAL MODELS}

\section{Ex vivo Anatomy}

While MR diffusion imaging can deliver useful information on the main fiber tracts in living brains (Jbabdi et al., 2015), ex vivo techniques are currently necessary to determine neuronal connectivity near or at synaptic resolution.

Light microscopy is limited in terms of imaging depth in scattering biological media. For this reason, serial mechanical sectioning followed by multiphoton imaging has been used in neuronal tracing (Ragan et al., 2012). Ex vivo tissue can also be made optically clear (Chung and Deisseroth, 2013; Renier et al., 2014) and imaged with light-sheet microscopy for nondestructive optical sectioning (Voie and Spelman, 1995; Power and Huisken, 2017; Mano et al., 2018). The resolution in light microscopy can be further improved by tissue expansion (Chen et al., 2015) and stochastic labeling of neurons (Livet et al., 2007).

Neuronal processes can have submicrometer diameters, with dendritic spine necks as small as $0.09 \mu \mathrm{m}$ in diameter (Bartlett and Banker, 1984; Arellano et al., 2007; Gipson and Olive, 2017) and follow complicated trajectories around each other and relative to the supporting astrocytes, vasculature, and microglia. Therefore, it is challenging to disentangle neuronal processes in densely labeled tissue using, e.g., the brainbow technique 
(Livet et al., 2007), even at the best-possible optical resolution. Thus, a complete characterization of all synaptic connections will ultimately require imaging the entire brain network via EM. Advances in volumetric EM techniques (Eberle and Zeidler, 2018; Yin et al., 2020) already permitted complete EM brain volumes from Drosophila (Zheng et al., 2018) and partial connectomes of larval zebrafish (Hildebrand et al., 2017) to come online, with substantial advances also made for the mammalian cortex (Motta et al., 2019).

PA imaging enables the structural interrogation of the brain at scales ranging from micro- to macroscopic. Either intrinsic or extrinsic photoabsorbers can generate image contrast. The main intrinsic absorbers in brain tissue are DNA, RNA, cytochrome, lipids, and water. Lipid concentrations vary in white matter $v s$. gray matter and between brain regions (Chavko et al., 1993). Since their photoacoustic spectra are masked in vivo by hemoglobin, lipid imaging is conducted ex vivo after brain perfusion ( $\mathrm{Li}$ L. et al., 2016). Mesoscale PAT imaging of entire mouse brains ex vivo has revealed structures such as the neocortex, cerebellum, cerebral aqueduct, corpus callosum, hypothalamus, hippocampus, inferior colliculus, olfactory bulb, and ventricles (Li L. et al., 2016; Olefir et al., 2019). Furthermore, dopaminergic neurons contain neuromelanin, an endogenous pigment that can be visualized ex vivo by photoacoustic tomography (Olefir et al., 2019).

PA microscopy, on the other hand, can resolve DNA/RNA (Yao et al., 2010), lipids (Wang et al., 2011), cytochromes (Zhang et al., 2013), hemoglobin (Yao et al., 2015), and melanin (Zhang et al., 2006) by selecting the appropriate wavelengths of laser illumination. Non-labeled brain tissue blocks measuring a couple of cubic millimeters were serially sectioned, scanned, and volumetrically reconstructed, allowing the mapping of nuclei positions within the blocks (Wong et al., 2017). PA prescans of unstained samples leveraging the intrinsic contrast of the previously mentioned photoabsorbers might provide a useful reference before executing complex fixation or staining protocols, leading to geometric tissue distortion.

Besides imaging the central nervous system, the spinal cord and peripheral nerves can also be accessed by PAI. Ex vivo PAT at an excitation wavelength of $1730 \mathrm{~nm}$ was employed to assess white-matter loss after spinal cord injury in adult rats (Wu et al., 2014). Myelin is the principal lipid constituent in nerves and was shown to produce distinctive PA microscopic signatures (Matthews et al., 2014). Nerves could also be visualized with hybrid PA/US systems in ex vivo preparations (Mari et al., 2015) and PAT in in vivo mouse models (Li R. et al., 2016). Imaging myelin in peripheral nerves might improve diagnostic accuracy if implemented in future clinical practice.

\section{In vivo Anatomy}

Microscopic brain imaging in vivo is dominated by multiphoton detection of fluorescent sensors. Besides imaging neurons, multiphoton imaging has also been used for the quantification of vascular architecture. In particular, measuring the microvasculature diameter during sensory stimulation can estimate the cerebral blood volume (CBV) and clarify its relationship to neuronal activation (Kong et al., 2015; Kim et al., 2019). Label-free PA microscopes can image brain microvasculature by detecting absorption signals from individual red blood cells (Kong et al., 2015; Kim et al., 2019). An integrated microscope combining reflection-mode photoacoustic, multiphoton, and second harmonic detection was used to image the complex $3 \mathrm{D}$ neurovascular system of mice in vivo (Song et al., 2016). Although oxygen metabolism was not quantified in this study, the ability to image neuronal activity at a subcellular level and simultaneously record hemodynamic parameters would prove valuable in elucidating the underlying mechanisms of neurovascular coupling (Song et al., 2016).

Photoacoustic tomography in vivo is dominated by vascular contrast due to high hemoglobin concentrations that mask other photoabsorbers. Brain structures such as the third ventricle, superior sagittal sinus, and cerebral bridging veins were visualized in vivo (Razansky et al., 2011; Burton et al., 2013; Olefir et al., 2016; Chen et al., 2017). The carotid arteries and Willis Circle could be imaged with PA by illuminating through the oral cavity (Lin et al., 2015), delivering data similar to what, e.g., time-of-flight MRI techniques provide.

Anatomical imaging with a hybrid PA/US system enabled the correction of skull-induced artifacts in mice, facilitating microvasculature visualization with a field-of-view covering the entire cortex (Estrada et al., 2018). Imaging brain vasculature in vivo can also be achieved optically with short-wave infrared imaging (Bruns et al., 2017; Carr et al., 2018) and US (Errico et al., 2015). Both of these techniques feature several centimeters in penetration depth (Macé et al., 2011; Bruns et al., 2017) and excellent performance in capturing flow dynamics. In this configuration, photoacoustics could contribute valuable data on oxygen metabolism.

\section{Ischemia}

Concerning pathological brain conditions, PA is naturally suited for studying brain ischemia due to its sensitivity to blood oxygenation. PAT systems lend themselves to labelfree detection of stroke by recording an asymmetry in blood oxygenation. Furthermore, areas of compromised perfusion in which brain tissue may still be viable, the so-called penumbra, can be identified (Kneipp et al., 2014). Both ORPAM and PAT were able to identify ischemia 3-5 min after onset. Hemorrhagic transformation could also be detected (Lv et al., 2020).

OR-PAM systems can be easily modified to enable localized photocoagulation by increasing the laser beams' energy, such that small vessels can be occluded with high precision, leading to local ischemia (Hu et al., 2011a; Liao et al., 2013; Lv et al., 2020). After photocoagulation, ischemia caused a breakdown of the $\mathrm{BBB}$, leading to extravasation of Evans Blue bound to albumin to visualize the extent of ischemia (Lv et al., 2020).

\section{Tumors}

The ability to visualize anatomical structures based on contrast derived from exogenous and endogenous photoabsorbers makes PA imaging a valuable tool for non-invasive neoplastic disease monitoring, including brain tumors. Volumetric imaging of glioblastoma in nude mice was demonstrated without contrast 
agents by exploiting differential contrast under illumination with 800 and $850 \mathrm{~nm}$. The authors also quantified tumor $\mathrm{O}_{2}$ saturation, which increased 15 days after inoculation, whereas the tissue became hypoxic 42 days after inoculation (Balasundaram et al., 2018). In another study, integrin expressing tumor cells were tagged with an IRDye800-based contrast agent while also assessing tumor $\mathrm{SO}_{2}$, which was $13 \%$ lower than in healthy brain tissue (Li M. et al., 2008).

Melanoma cells offer strong intrinsic contrast for PAI. Secondary melanoma brain tumors were monitored noninvasively with a reflection-mode PAM in small rodents (Staley et al., 2010). Circulating melanoma cells could be imaged in the brain of mice perfused with artificial cerebrospinal fluid, which was possible because melanoma cells naturally contain the highly absorbing pigment melanin. Still, high hemoglobin absorption prevented a transfer of the technique to in vivo detection (Deán-Ben et al., 2020). In addition to cell visualization, it is possible to successfully manipulate circulating B16F10 mouse melanoma cells with acoustic and photoacoustic pressure waves. Circulating cells in lymphatic vessels were trapped, focused, and redirected, showcasing how this method might be used for the non-invasive diagnosis of neoplastic disease (Galanzha et al., 2016). Identification of circulating melanoma cells in the superficial venous system of human subjects was also achieved with a PAT system. However, more extensive studies are needed to determine the value of including this imaging method in the staging of this neoplastic disease (Hai et al., 2020).

\section{Neurodegenerative Diseases}

Amyloid plaque deposition is a hallmark of Alzheimer's disease. Inflammatory processes and abnormal oxygen metabolism also play an important role in neurodegenerative processes and may be accessed by molecular imaging.

Positron emission tomography is considered the gold standard in Alzheimer's plaque and inflammation imaging. Despite recent developments in simultaneous PET/MR acquisition, molecular information is limited to low spatial resolution in the $0.5-1$ millimeter range (Zhang X. Y. et al., 2017). Optical imaging in preclinical settings has been facilitated by the development of NIR contrast agents targeting amyloid plaques (Tong et al., 2015). The plaques were visualized with an OR-PAM system after i.v. congo red injection, which is a classical photoabsorbing stain for amyloid. Plaque position, morphology, and relation to vasculature were imaged both in vitro and in vivo through a glass window. Again for this application, multispectral acquisition allowed blood vessels to be simultaneously imaged without contrast agent injection (Hu et al., 2009).

Inflammatory processes can be imaged with PA by using macrophage targeting probes such as CDnir7 (Kang et al., 2014). Alzheimer's disease's inflammatory component was quantified non-invasively in vivo using PAT detection after CDnir7 administration (Park et al., 2019).

Cerebral metabolic dysfunction has been shown to accompany plaque deposition in AD (de la Monte and Tong, 2014). PAT investigation of $\operatorname{arcA} \beta$ mice showed a decrease in the cerebral metabolic rate of oxygen $\left(\mathrm{CMRO}_{2}\right)$, while the oxygen extraction fraction (OEF) remained unchanged in control animals (Ni et al., 2018).

\section{IMAGING OF BRAIN HEMODYNAMICS}

A transient increase in neuronal activity leads to higher energetic demands in spatially confined regions of the brain. However, neuronal tissue does not possess significant energy stores (Magistretti and Pellerin, 1999; Shulman et al., 2001). Instead, nearby vessels dilate to ensure an adequate supply of glucose and oxygen, which are metabolized locally in neurons. This coupling of neuronal activity to cerebral blood flow (Huneau et al., 2015) is referred to as neurovascular coupling. Consequently, neuronal activation events can be inferred from observed hemodynamic signal changes via an estimate of a transfer function, the so-called hemodynamic response function (HRF) (Peng et al., 2019).

Functional imaging of the brain based on the neurovascular coupling is thus possible with essentially any imaging modality that can detect a transient change in blood volume, blood flow, or blood oxygenation. Here, we highlight the strengths and limitations of MRI, US, and optical imaging modalities to describe how PA can complement these modalities based on its sensitivity to blood flow dynamics and hemoglobin oxygenation.

Hemodynamic signal changes are famously imaged by fMRI, employing the so-called Blood Oxygenation Level Dependent (BOLD) contrast (Ogawa et al., 1990, 1992). BOLD is sensitive to changes in the concentration and oxygenation state of hemoglobin. The precise physiological mechanisms underpinning the BOLD contrast are, however, still not fully understood. Multimodal imaging systems which co-record fMRI data with PET (Aiello et al., 2016), EEG (Moeller et al., 2020), NIRS (Scarapicchia et al., 2017), and fluorescence imaging (Schwalm et al., 2017; Lake et al., 2020) are thus being employed to cross-validate the fMRI signals.

For preclinical experiments, fMRI is limited mainly to anesthetized measurements, although protocols for imaging awake mice (Chen et al., 2020), rats (Chang et al., 2016), and non-human primates (Chen et al., 2012) have been described. Temporal resolution for fMRI is limited to one imaging volume per second though trade-offs in spatial resolution enable line scanning at $10-20 \mathrm{~Hz}$ (Yu et al., 2014; Albers et al., 2018). Due to its non-invasiveness, fMRI has been extensively used to map functionally connected brain regions in humans (Maknojia et al., 2019).

Functional ultrasound interrogation and manipulation of brain networks have been proposed to overcome some of fMRI's shortcomings in specificity, spatiotemporal resolution, and portability (Rabut et al., 2020). Cortical and deep thalamic sensory responses to stimulation were characterized by dopplerultrasound imaging (Macé et al., 2011). Single-trial functional brain responses to stimulation in unconstrained behaving monkeys were recorded by ultrafast Doppler (Dizeux et al., 2019). Moreover, implantable ultrasound transducers combined with EEG allow more natural behavioral experiments to be carried out in mobile behaving rats (Sieu et al., 2015). A drawback for this modality derives from the fact that fUS imaging in human 
subjects is only possible in infancy before fontanelle calcification (Demene et al., 2017) or after skull removal for brain surgery (Imbault et al., 2017).

Optical imaging in the short wave infrared (SWIR), 1 to $2 \mu \mathrm{m}$ in wavelength, facilitates hemodynamic measurements at depths greater than multiphoton microscopy. Highly detailed representations of the brain's vasculature have been acquired after injection of indocyanine green (ICG) (Carr et al., 2018; Byrd et al., 2019), quantum dots (Bruns et al., 2017), and organic fluorophores (Wan et al., 2018), enabling the generation of detailed blood flow maps (Bruns et al., 2017). While this technique may enable deep brain functional imaging, further improvements in detector technology and molecular imaging sensors are necessary before widespread implementation.

Multispectral PAI can enhance the information gained from the techniques mentioned above through the non-invasive quantification of oxygenated ( $\mathrm{HbO})$ and deoxygenated $(\mathrm{HbR})$ hemoglobin, total hemoglobin (HbT), and cerebral blood flow (CBF). From these parameters, the oxygen extraction fraction $(\mathrm{OEF})$ and the cerebral metabolic rate of oxygen $\left(\mathrm{CMRO}_{2}\right)$ can be calculated. Photoacoustic microscopy has been used to investigate the hemodynamic coupling at the microvasculature scale by $\mathrm{OEF}$ and $\mathrm{CMRO}_{2}$ estimation (Liao et al., 2010, 2012; Yao et al., 2015; Cao et al., 2017). OR-PAM has also made important contributions to our understanding of the physiological underpinnings of the BOLD signal in fMRI. An increase in HbR in cerebral arterioles was observed hundreds of milliseconds after electrical paw stimulation, which likely corresponds to the initial decrease in BOLD signal commonly referred to as the 'initial dip' (Liao et al., 2012).

Although a hybrid multiphoton and photoacoustic microscope has been presented (Song et al., 2016), this was mainly used for structural imaging. Simultaneous recordings of genetically encoded fluorescent calcium indicators and PAI of hemodynamic parameters could shed light on individual contributions of neurons and glial cells to CBF increases (Muñoz et al., 2015). To achieve these results, OR-PAM techniques usually still necessitate scalp and sometimes also skull removal and are limited to a penetration depth of about a millimeter (Hsu et al., 2018).

Because of the limitations of OR-PAM, tomographic PAI techniques have also been employed for imaging neuronal activation via neurovascular coupling. Photoacoustic signal enhancements localized to large cortical blood vessels in response to sensory stimulation have been reported (Wang et al., 2003a). In addition to cortical responses to sensory stimulation (Zhang et al., 2018; Olefir et al., 2019), thalamic signals deeper in the brain also seemed to be detectable by PAT (Zhang et al., 2018). Brain hemodynamic imaging with a PAT system revealed functional brain networks in a centrally situated coronal brain slice in mice (Olefir et al., 2019). The whole mouse brain can theoretically be captured by this imaging technique, though covering the entire brain volume requires moving either the transducer array (Wang et al., 2003b; Zhang et al., 2018) or translating the subject (Burton et al., 2013; Olefir et al., 2016).
Volumetric PA acquisition for whole-brain imaging is desirable and can be achieved by using an ultrasound detector array mounted on a hemispherical surface (Gottschalk et al., 2019b). Light is delivered via an optical fiber bundle situated in a central opening in the transducer array. Such a configuration allows for $100 \mathrm{~Hz}$ volumetric imaging, with variable wavelengths on a per laser pulse basis, with a spatial resolution of 175 $\mu \mathrm{m}$ (Chen et al., 2017). Practically, spectral unmixing of $\mathrm{HbO}$, $\mathrm{HbR}$, and $\mathrm{HbT}$ require data acquisition after illumination at $506,540,560,575$, and $585 \mathrm{~nm}$ wavelengths, which limits the effective temporal resolution after unmixing to 20 brain volumes per second. After electrical paw stimulation, 31.4, -3.5, and 6.6 percentage changes in $\mathrm{HbO}, \mathrm{HbR}$, and $\mathrm{HbT}$ relative to baseline were observed (Mc Larney et al., 2020).

Hemodynamic PAI in awake, behaving rats has also been reported. 3D data acquisition was performed with an implantable miniaturized three-layer transducer array providing a field-ofview that covered the entire brain. After presenting optical stimuli, CBV was quantified in the visual cortices and the superior sagittal sinus showing a perfusion increase after stimulation (Tang et al., 2016). However, challenges in miniaturization remain for this approach and implantable microscopes or mobile ultrasound detectors represent cheaper and more practical options.

\section{Epilepsy}

Hemodynamic imaging also lends itself to the study of pathologies that induce massive neuronal activation followed by a hemodynamic response such as epileptic seizures. Proof of principle work in this pathology has been performed with spherical arrays in rats (Wang et al., 2012) while seizures in awake animals were imaged using portable sensor arrays (Tang et al., 2015). PAT helped to characterize the spatial spread of pharmacologically induced seizure activity in the mouse (Zhang et al., 2018) and could also discern patterns related to seizure activity in thalamic regions beyond the reach of optical imaging (Gottschalk et al., 2017). Tissue perfusion changes showing increased blood flow in seizure foci measured with PA are also in agreement with optical imaging spectroscopy studies (Harris et al., 2014).

As accessible as the neurovascular coupling is for many imaging methods, it only provides a rough estimation of the metabolic activity in a given brain region. Thus, it is probably biased toward energy-intense presynaptic neurotransmitter recycling (Logothetis, 2008; Gauthier and Fan, 2019), while it can not differentiate between excitatory or inhibitory neurotransmission. This contrast mechanism can also not resolve individual cells or the timing of action potentials, which can be accessible with molecular contrast agents.

\section{MOLECULAR CONTRAST FOR PHOTOACOUSTIC NEUROIMAGING}

Molecular bioimaging aims to non-invasively map biological structures and monitor cellular processes at the molecular level. Molecular contrast agents convert a specific molecular 
structure or process of interest into an appropriate signal for a given imaging method, which can otherwise not obtain this information. If the signals from the molecular contrast agents can be made sparse, the molecular information can also be localized at a resolution that exceeds that dictated by the imaging device's point-spread-function (Betzig et al., 2006). In this regard, molecular contrast agents amplify and magnify molecular information.

This section addresses the most promising contrast mechanisms currently available for PAI in neuroscience.

\section{Desired Properties of Molecular Contrast Agents for PA}

For PA imaging, the building blocks for contrast agents ideally fulfill the following set of photophysical properties: a large molar extinction coefficient at wavelengths at which tissue absorbance is relatively low such as in the near-infrared window, a distinct absorption peak to facilitate spectral unmixing, a low quantum yield for maximum light conversion to thermal energy via nonradiative decay, and a high photostability (Weber et al., 2016).

Concerning the interaction with the biological tissues of interest, a molecular contrast agent should also possess high stability in biological media such as blood and cerebrospinal fluid, low toxicity, and immunogenicity. For neuroimaging, the contrast agent's size and charge should also enable permeability through the blood-brain-barrier (BBB) (Milej et al., 2017), such that techniques to open the $\mathrm{BBB}$ do not have to be employed.

There is a wide variety of contrast mechanisms applicable in preclinical neuroimaging. It is thus useful to differentiate between the following molecular contrast agent classes with respect to which molecular state or process they indicate: (i) targetable labels that can map the biodistribution of a target structure, (ii) turn-on probes, whose signal can be irreversibly activated by a molecular interaction, and (iii) reversible sensors, which can dynamically adopt different signaling states in response to an analyte of interest (Figure 2, columns).

Based on their composition, we can also differentiate synthetic from semi-genetic or fully genetic contrast agents (Figure 2, rows).

Synthetic agents are produced through chemical and nanotechnological methods. Semi-genetic contrast agents have a genetically encoded component that interacts with at least one synthetic component. In distinction, genetic contrast agents are generated via genetic programming of a biosynthetic pathway within a cellular (or cell-free) biomolecular machinery, which may require endogenous co-factors.

The molecular contrast agent type has, for instance, implications for the delivery method that can be used. Options for delivering contrast agents to the brain include intravenous, intrathecal, intracerebroventricular, or intracerebral injections.

For intravenous delivery, a prolonged circulation time may be desired for imaging blood flow and contrast agent accumulation in the target tissue.

To overcome the intact BBB, high lipid solubility and a particle size under $1 \mathrm{~nm}$ are advantageous (Abbott and Romero, 1996; Pardridge, 2005). In neoplastic disease, BBB disruption allows the passage of larger particles up to $100 \mathrm{~nm}$ (Houston et al., 2020). Transport processes across the BBB can be exploited by incorporating suitable modifications that enable receptormediated transcytosis (Pulgar, 2018).

The BBB can also be temporarily opened using focused ultrasound, thus increasing the spectrum of compounds that can be efficiently delivered to the brain (Chen et al., 2019).

An intracranial injection is an alternative option for the direct delivery of dyes, therapeutics, or viruses to the brain. This method's limitations are that only a small volume can be administered (usually around 1-2 $\mu \mathrm{L}$ ) at low injection rates, usually not exceeding $0.1 \mu \mathrm{L} / \mathrm{min}$ to avoid damaging the brain (Cetin et al., 2006). Alternatively, surgically implanted osmotic pumps can dispense defined volumes over longer periods (Sepúlveda et al., 2012).

Whereas synthetic agents have to be delivered to the tissue of interest and then targeted to extra- or intracellular structures, genetically encoded contrast agents have the advantage that they can be produced directly in genetically defined cells conditioned on specific cellular states. Transferring the necessary genetic information can be achieved by germline transgenesis or via the administration of viral (Sung and Kim, 2019) or non-viral (O’Brien and Lummis, 2006) DNA or RNA delivery vehicles.

Gene expression via viral transduction is preferred in vivo and can be achieved through interstitial injection of neurotropic viruses or systemic vector administration (Foust et al., 2009; Manfredsson et al., 2009; Challis et al., 2019). Popular vectors include Lentiviruses, Herpes Simplex, or Adeno-associated viruses (Cetin et al., 2006; Challis et al., 2019; Ingusci et al., 2019). The selective use of gene promoters allows for an increasing number of neuronal or glial cell types to be specifically targeted. After infection, the contrast agent DNA sequence can be integrated into the host genome (Vargas et al., 2016), or it persists as an episome in the nucleus (Haggerty et al., 2020). Regardless of DNA integration, because neurons do not divide, long-term contrast agent expression can be achieved.

Besides, the knowledge that different neurotropic viruses have a preferred direction of transport within infected neurons can be exploited to identify or target the pre- or postsynaptic connections of a neuronal population of interest (Saleeba et al., 2019).

\section{Intravascular Contrast Agents}

The specific biodistribution of contrast agents in predefined compartments of the body can reveal local anatomical and functional aspects. Examples include "blood pool agents" that are constrained to the vascular system, nanomaterials that accumulate in tumors or post-stroke tissue due to enhanced permeability and retention effects (PRE) (Nel et al., 2017), or leakiness of the BBB.

At a finer scale, information can be gained from targetable labels that can be directed to specific cells via surface affinities or selective cellular uptake (see next section).

Many synthetic photoabsorbers can serve as blood-pool agents and as the building blocks for targetable agents in PAI: organic contrast agents (e.g., organic dyes), conjugated 


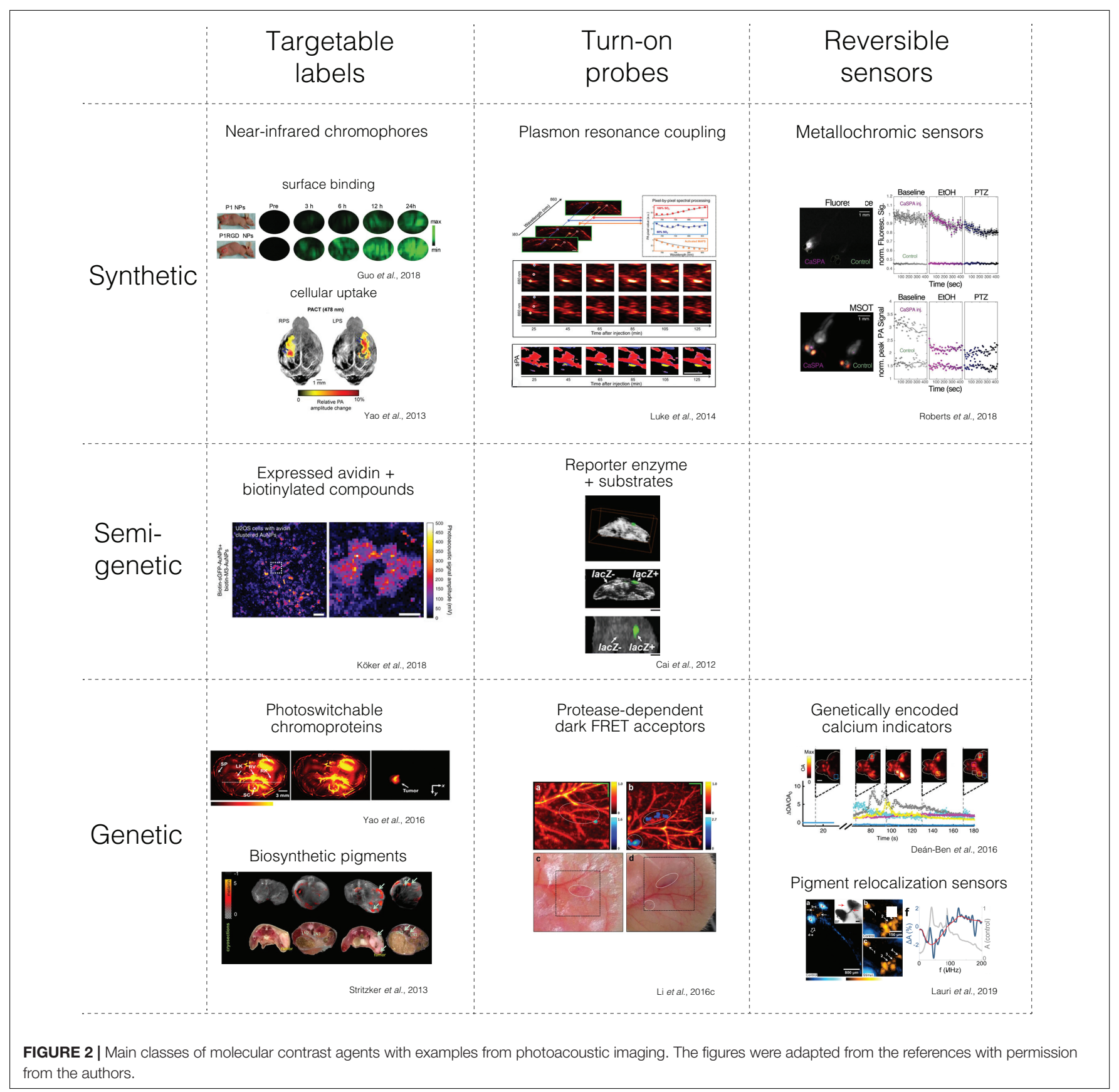

polymer nanoparticles, and other inorganic nanostructures (e.g., carbon nanoshells).

Small molecular dyes can visualize blood vessels and enable perfusion measurements in healthy brains and brain tumors (Burton et al., 2013). Strong photoabsorbers such as black paramagnetic polyethylene microspheres (Luís Dean-Ben and Razansky, 2018) or oil-dissolved IR-780 dye droplets (Zhang P. et al., 2019) were used for localization microscopy with photoacoustics to go beyond the diffraction limit. Applying these techniques to PA angiography enables high-resolution maps of brain vasculature to be acquired in vivo (Zhang P. et al., 2019).
Advances in material science have led to the creation of functionalized synthetic nanoprobes, which can be tuned for high absorption in a defined spectral range, exhibit resistance to photobleaching, and be functionalized with the addition of dyes or drugs. Most synthetic nanoparticles developed for photoacoustic applications have been employed for studying brain tumors: noble metal nanoparticles (Shang et al., 2017), copper nanoparticles (Wu et al., 2018; Zhang H. et al., 2019), carbon nanorods (Qian et al., 2018), iron-based nanoparticles (Zhou et al., 2018), MoS2 nanosheets (Chen et al., 2016). Nanoparticles that enable multimodal imaging have been a focus of recent research: PA/MRI/Raman (Kircher et al., 2012), 
PA/MRI/PET (Fan et al., 2014), PA/MRI (Zhu et al., 2015; Qiao et al., 2018). Circulating dyes and nanoparticles have been shown to accumulate in brain tumors due to a disruption of the $\mathrm{BBB}$ and PRE (Kircher et al., 2012; Burton et al., 2013; Fan et al., 2015; Neuschmelting et al., 2018). However, these contrast agents are rapidly cleared from the bloodstream by the reticuloendothelial system and the liver.

\section{Targetable Labels}

\section{Synthetic Targetable Labels}

Strategies to achieve specific targeting of cellular structures as opposed to just relying on PRE include contrast agents binding to endothelial tumor cells (Ray et al., 2011), transferrin receptors (Zhu et al., 2017), adenosine receptors (Liu et al., 2019), and integrin (Guo et al., 2018). Additionally, the photoabsorbing properties of PA contrast agents make them suited for hyperthermia treatment in glioblastoma (Manivasagan et al., 2017; Zhu et al., 2017; Balasundaram et al., 2018; Guo et al., 2018; Liu et al., 2019) (Figure 2, upper left segment).

A good example for a targeted cellular uptake is 2-NBDG, a fluorescent 2-deoxyglucose variant, which in analogy to the wellestablished method for PET, can be internalized preferentially into cells that have an increased energy demand. Once taken up by the glucose transporter, the glucose analog is phosphorylated by cellular kinases such that it cannot leave the cell. Using this compound in PA imaging, it was possible to visualize increases in brain glucose metabolism after sensory stimulation and quantify hemodynamic brain responses in the same experiment using two excitation wavelengths (Yao et al., 2013).

Contrast agents may also be targeted to selective cells ex vivo, which are then injected into an organ(ism). In this mode, the cell itself can be considered the contrast agent, and its interactions in the tissue can be monitored. Mesenchymal stem cells labeled with Prussian blue were injected intravenously and used to monitor the extent of the healing process after traumatic brain injury (Li et al., 2018). Similarly, tumors can be targeted for imaging using nanoparticle-labeled mesenchymal stem cells (Qiao et al., 2018). However, synthetic labels have the disadvantage that they are rapidly diluted upon cell divisions, and that contrast will also persist even if the cell has already died, shortcomings that can be alleviated with genetic labels.

\section{Semi- and Fully Genetic Targetable Labels}

Whereas fully-synthetic targetable labels depend on a selective targeting mechanism that has to exploit a particular cell surface property or a selective uptake mechanism, genetic targeting mechanisms can provide selectivity by tapping into the specific genetic program of particular cells (Figure 2, middle and bottom row).

The genetic component can be stably expressed in germline transgenic animal models such that the contrast can be imaged without further intervention. Alternatively, the genetic information for encoding the label can also be introduced to an organism via (non-)viral carriers or via delivering genetically modified cells. In all cases, the contrast can be made dependent on the genetic program of selective cells, e.g., by using specific promoters. The genetic label also propagates to daughter cells without loss and is also selective for viable cells as their expression relies on an intact cellular metabolism.

Semi-genetic targeting of a synthetic label can, for instance, be achieved by expressing an affinity handle, e.g., on the cell surface. In one such system, biotinylated gold-nanoparticles were bound to avidin expressing cells in culture (Köker et al., 2018) or lung cancer cells in a rodent model (Lee et al., 2018) and detected by PA.

As pertains to fully genetic labels, fluorescent proteins such as mCherry have been shown to give rise to photoacoustic contrast (Razansky et al., 2009). However, the absorbance peak in the visible spectrum competes with strong photoabsorbance from hemoglobin in vivo and thus limited PA imaging to zebrafish and fly pupae. Moreover, chromoproteins consisting of chromophores built only from aminoacids via cyclization reaction have generally low photostability, as is the case for GFP derivatives.

In comparison, chromoproteins, which bind small biosynthetic chromophores such as biliverdin, exhibit higher photostability and absorbance peaks that reach further into the near-infrared window than red proteins such as E2-Crimson, mNeptune, mKate2, eqFP670, and TagRFP65, resulting in superior photoacoustic signals (Filonov et al., 2012). For instance, IRFP expressing U-87 MG cells could be detected after cerebral injection into nude mice (Deliolanis et al., 2014).

Another powerful strategy to increase the contrast of fullygenetic labels is to use reversibly switchable fluorescent proteins (RSFP), which can be switched into a non-fluorescent dark state by laser illumination accompanied by a shift of the absorption spectrum (photochromism) (Figure 2, lower left segment). This photophysical property allows for photocontrol of distinct timevarying signals (Marriott et al., 2008), which can be temporally unmixed reliably from each other and from strong static background absorbers such as hemoglobin in PAT even if they are spectrally overlapping (Deán-Ben et al., 2015b; Stiel et al., 2015). By expressing switchable bacteriophytochrome (BphPs) proteins with absorbance spectra in the near-infrared, tumor cells could be differentiated from background in mouse brain $\sim 3 \mathrm{~mm}$ beneath the scalp surface subdiffraction imaging of individual tumor cells (Yao et al., 2016). Expression of BphPs also allowed for the segmentation of tumor cells from subcutaneous tumor vasculature in vivo (Märk et al., 2018) and differentiating bacteria injected in tissue (Chee et al., 2018). Expression of selected truncated BphP variants optimized for extinction coefficients, switching kinetics, and reduced photofatigue allowed for the segmentation of as few as 500 cells/ul Jurkat $\mathrm{T}$ lymphocytes after subcutaneous injection into mice via classification of a set of features extracted from the photoswitching signal trajectories (Mishra et al., 2020).

In distinction to chromoproteins, biosynthetic pigments, i.e., small molecular dyes synthesized by enzymatic processes, can have a high photostability. Each translated enzyme performs many rounds of catalysis, producing numerous photoabsorbers, thus leading to a substantial amplification factor of the genetically defined process.

Melanin is a polymer derived from the amino acid tyrosine as a substrate for the enzyme tyrosinase. Melanin offers excellent 
contrast in PA imaging, as is well-known from skin imaging of melanin-containing nevi and ephelides. Early on, tyrosinase has thus been used as a reporter enzyme for photoacoustics (Krumholz et al., 2011; Stritzker et al., 2013). However, Melanin precursors have cytotoxic properties if they are not sequestered in melanosomes, membranous compartments found in specialized cells (Raposo and Marks, 2007). A Tet-On inducible tyrosinase reporter-gene system was expressed in tumor cells, which were implanted in vivo. Upon doxycycline administration, melanin production could be initiated, and this process could be visualized in vivo with PA imaging (Paproski et al., 2014). The inducible gene expression can control the duration and toxicity to some degree, but more delicate cells such as neurons may still be substantially compromised. Another solution was found by expressing self-assembling nanocompartments that can accept guest molecules to their lumen and thus sequester enzymatic processes. Specifically, tyrosinase was targeted to the inner surface of so-called encapsulins from the bacterium $M$. xanthus, which assemble to semi-permeable icosahedra that would allow the substrate tyrosine but sequester the toxic melanin polymer. Consequently, melanin production was confined to the lumen of the engineered nanomelanosomes, producing strong PAT signal but shielding the cells from toxicity-induced damage produced by non-encapsulated tyrosinase (Sigmund et al., 2018). It will be exciting to see combinations of these labels with recently developed gene reporters for US (Farhadi et al., 2019).

\section{Turn-On Probes}

An example of fully synthetic turn-on PA contrast agents are $\mathrm{BBB}$-permeable aniline compounds that strongly increase absorbance and thus PA signal around $750 \mathrm{~nm}$ upon chelation with $\mathrm{Cu}^{2+}$ and may thus be useful for imaging copper accumulation in brains that may be correlated with amyloid plaque formation (Wang et al., 2019). Furthermore, gold nanoparticles conjugated to antibodies have been shown to change their PA spectra upon target binding due to plasmon resonance coupling (Luke et al., 2014).

One of the first semi-genetic turn-on mechanisms adapted for PA imaging is the well-known reporter enzyme $\beta$-galactosidase, which converts the soluble synthetic substrate $\mathrm{X}$-gal into a blue precipitate. Delivery of the $\mathrm{X}$-gal substrate to $\beta$-galactosidaseexpressing cells in culture was shown to generate PA contrast from the resulting precipitate upon $650 \mathrm{~nm}$ illumination (Li et al., 2007). Simultaneous detection of the microvasculature and lacZ labeled tumor cells via AR-PAM imaging was useful in identifying tumor feeding vessels ( $\mathrm{Li}$ L. et al., 2008). Individual LacZ labeled cells could be imaged with an OR-PAM setup showing intracellular contrast agent distribution, and PAT methods were able to detect LacZ labeled cells up to a depth of $5 \mathrm{~cm}$ in biological tissue (Cai et al., 2012).

Förster resonance energy transfer (FRET) entails energy transfer from a donor fluorophore to an acceptor chromophore, which can also alter the corresponding PA signals (Wang and Wang, 2012; Wang et al., 2013) (Figure 2, bottom of middle column). An activatable PA reporter for protease activity was explored in vitro using genetically expressed chromoproteins with low QY as a dark FRET acceptor. The authors expressed ultramarine variants linked to EGFP as a FRET donor via a caspase-3 cleavage site ( $\mathrm{Li}$ Y. et al., 2016). When caspase-3 activity was induced in mammalian cell culture, an increase in EGFP fluorescence was observed due to the loss of FRET upon proteolytic separation of the FRET pair. When FRET-pairs were purified and measured by PA without and with proteolytic cleavage of the linker, a decrease in the PA signal was observed consistent with an increase of donor fluorescence. Modulation of FRET from a dark acceptor could be an interesting PA reporter mechanism, especially if genetically controlled FRET donors with high QY in the near-infrared could be developed.

\section{Dynamic Sensors}

As discussed above, volumetric PA imaging is a scanless technique that can pick up signals with higher temporal resolution and from deeper tissue layers than multiphoton techniques. Thus, it is an attractive method for mapping dynamic and distributed brain processes. As laid out above, PAI can map neurovascular coupling dynamics at adequate temporal sampling rates and over large portions of the brain.

However, it is desirable to obtain more specific information about the cellular and molecular identity and temporal profile of neuronal activity.

Patch-clamp measurements of neurons naturally provide the best spatial and temporal resolution for characterizing neuronal electrical activity (Petersen, 2017), but it is often valuable to capture the propagation of charge fluxes over many cells of specific identities.

Imaging electrical events in neurons was first achieved with electrochromic sensors, in which membrane potentials cause changes in absorption (Tasaki et al., 1968; Ross et al., 1977; Grinvald et al., 1981; Grinvald and Hildesheim, 2004). Despite the low signal changes of usually less than $1 \%$, impressive imaging results allowed millisecond activation maps resolving individual columns in the visual cortex, a highly influential finding for the field of visual perception (Sharon and Grinvald, 2002).

While synthetic voltage dyes also tend to stain glia, genetically encoded voltage-sensitive indicators (GEVIs) can be targeted to selective neuronal types. Several types of GEVIs have been realized based on fusions to voltage-sensing domains or rhodopsins, which can be read out by FRET or QY changes. They have become brighter and increasingly red-shifted, enabling the study of subcellular features of the action potential waveform (Xu et al., 2017; Bando et al., 2019; Panzera and Hoppa, 2019). Challenges persist in applying this technology in vivo as heavily convoluted neuronal cells make it difficult to accurately record subcellular signals with sufficient speed and spatial resolution to avoid partial volume effects. Despite these technical challenges, all-optical electrophysiological (optophysiological) studies have begun to provide insight into the circuit activity underpinning behavior (Adam et al., 2019).

There have been initial attempts to pick up voltage changes by PA via fluorescence quenching of voltage-dependent dyes, which should increase PA intensity (Zhang $H$. K. et al., 2017). The fluorescent voltage sensor dipicrylamine was imaged with OR-PAM in HEK cells in vitro and PAT in vivo after administration of chemoconvulsants (Rao et al., 2017). 
Similarly, the voltage sensor VSD IR780 perchlorate was imaged in vivo after seizure induction (Kang et al., 2019). While VSD fluorescence changes were used as a positive control in these studies, in vivo ground-truth electrophysiology during more physiological stimulation paradigms would be desirable to assess these contrast agents' potential.

Given the small signal change and the limited concentration and slow replacement of photobleached voltage sensors in the cell membrane, PA detection of voltage changes will remain challenging but very desirable to advance fast volumetric voltage imaging.

Calcium transients are too slow to identify the precise timing of action potentials, such as synchronicity between AP trains (Kwan and Dan, 2012; Hoang et al., 2020). However, calcium dynamics lie within the temporal resolution of many current imaging methods, and estimating action potentials by deconvolution is possible under certain conditions (Pachitariu et al., 2018; Hoang et al., 2020). Furthermore, intracellular calcium concentrations can change by two orders of magnitude in response to neuronal spiking (Schiller et al., 1995; Ali and Kwan, 2019) and can be measured in the cytosol and nucleus of the cell, where higher concentrations of calcium sensors can be achieved than possible for membrane-bound voltage sensors.

Volumetric PAI at current frame rates up to $100 \mathrm{fps}$ is better suited for recording $\mathrm{Ca}^{2+}$ transients than action potentials due to the slower kinetics of calcium sensors (Chen et al., 2013).

Canonical calcium sensors include synthetic dyes such as Oregon Green 488 Bapta-1 AM-ester, which can reach the cytosol, where endogenous esterases cleave the AM-ester to activate and trap the sensor inside cells (Tsien, 1980, 1981; Stosiek et al., 2003). As for genetically encoded calcium indicators (GECIs), a design based on GFP fused to calcium-binding protein $\mathrm{CaM}$, and its binding peptide (GCaMP) has been substantially optimized over two decades to yield robust tools for routine measurements (Miyawaki et al., 1997; Chen et al., 2013; Yang et al., 2018). Furthermore, ratiometric calcium sensors have been built by crosslinking FRET pairs via a calcium-triggered contractile element from muscle fibers (Mank et al., 2008; TrigoMourino et al., 2019).

As a step to enable intracellular calcium imaging with photoacoustics, a synthetic calcium sensor for photoacoustics (CaSPA) was recently designed to selectively respond with a robust blue-shift of the spectrum (metallochromism) upon calcium binding while minimizing quantum yield and ensuring high photobleaching resistance (Roberts et al., 2018) (Figure 2, upper right segment). Due to its esterified BAPTA moiety (Tsien, 1981), the compound could be delivered into cells, organoids, and into zebrafish and exhibited high selectivity. CaSPA is based on a semi-cyanine chromophore that can be modified to red-shift the absorbance spectrum, which is an obvious next goal to separate it from the brain activity-dependent hemoglobin absorbance in the visible range.

It could also be recently shown that GCaMP can be detected by PAI, which was chosen because it exhibits a strong change in absorbance instead of calcium sensors that work by a change in quantum yield (Deán-Ben et al., 2016) (Figure 2, lower right segment). In proof-of-principle experiments, simultaneous fluorescence, and photoacoustic recordings were conducted in GCaMP-expressing zebrafish larvae and ex vivo adult zebrafish brains using chemical calcium triggers. These results have been corroborated by an ex vivo murine brain preparation showing signal changes from GCaMP6f in response to an activating chemoconvulsant (Gottschalk et al., 2019a).

As with synthetic calcium sensors, one obvious path for improvement will be to develop red-shifted calcium-dependent chromoproteins, e.g., BphPs, as detailed above. For example, NIR-gecol exhibits absorbance and emission maxima at 678 and $704 \mathrm{~nm}$, respectively (Qian et al., 2019). However, the protein is only moderately metallochromic, and in vivo paw stimulation experiments resulted in only a $0.3 \%$ fluorescence signal decrease in the somatosensory cortex of mice (Qian et al., 2019), while no photoacoustic signal changes were shown. Another recently developed genetically encoded $\mathrm{Ca}^{2+}$ sensor, iGECI, is a NIR Förster resonance energy transfer (FRET)-based calcium indicator with a fixed maximum absorbance at $670 \mathrm{~nm}$. In vivo imaging measurements yielded a negative $3 \%$ change in fluorescence after stimulation. The authors report simultaneous iGECI fluorescence and PA imaging of hemodynamics but no direct PA detection because PA signals are likely too small to exceed the noise (Shemetov et al., 2020). Further, NIR $\mathrm{Ca}^{2+}$ sensors like GAF-CaMP2 have also been reported to have modest absorbance change and have only been validated in cell culture (Subach et al., 2019).

None of the constructs listed above has the necessary properties for successful application in PA imaging of calcium transients in the NIR. One way to mitigate this problem might be to develop a reversibly switchable calcium sensor, applying some of the strategies illustrated in the section on reversibly switchable photochromic proteins above, and use more complex signal acquisition and data processing schemes to derive calcium dynamics. Research in this area is intense as, apart from the application in PA imaging, NIR $\mathrm{Ca}^{2+}$ sensors would be valuable tools for multiplexing with other visible range $\mathrm{Ca}^{2+}$ reporters and optogenetic actuators, enabling more complex neurophysiological experiments. $\mathrm{Ca}^{2+}$ imaging with $\mathrm{PA}$ could also benefit from progress made in image acquisition hardware and new computational strategies for spectral unmixing and image reconstruction.

A helpful step toward developing such a sensor would include the standardization of PA imaging setups and protocols for high-throughput screening of biosensor candidates (Hofmann et al., 2019).

A central signal transduction pathway in neurons, for which molecular sensors are desired, are GPCRs. GPCRs are a large family of cell surface receptors that interact with a wide variety of external signals, which regulate a myriad of cellular functions (Gurevich and Gurevich, 2019). This function makes them one of the most attractive targets for pharmaceutical intervention. Binding of an extracellular ligand to a GPCR results in G-protein activation, followed by signaling through the cAMP or phosphatidylinositol pathway, which releases intracellular calcium.

This signal transduction chain was used to generate GPCRreporter cells that express a specific GPCR together with a 
calcium indicator, such that a calcium-induced fluorescence signal change answered the presence of a specific ligand. By implanting these whole-cell sensors into the brain of mice, dopamine and norepinephrine release could be monitored via fluorescence (Muller et al., 2014).

Direct fluorescence sensors for GPCR activity were designed in analogy to GCaMP by fusing circularly permuted fluorescent proteins to GPCR, which translate conformational changes in the GPCR to the fluorescent protein, altering the fluorescent signals (Patriarchi et al., 2018; Sun et al., 2018). Although these sensor mechanisms may, in principle, also be accessible by photoacoustics, the current wavelength range is far from optimal, as discussed above.

Recently, a different PA contrast mechanism was introduced based on the reversible relocalization of biosynthetic pigments (Lauri et al., 2019). To turn the static pigments into dynamic contrast changes, the authors took inspiration from cuttlefish, which can change their skin's photoabsorbing patterns by rapidly contracting or dispersing pigment-filled compartments within specialized chromatophore cells via GPCR-induced calcium influxes. As a biomimetic analogon, cells containing melaninfilled melanosomes from Xenopus laevis were transplanted into the midbrain of juvenile zebrafish. The strong PA contrast enabled RSOM (an AR-PAM implementation) to resolve the contraction of individual melanophore cells in response to ligand-induced GPCR activity. The pigments' contraction and dispersion could also be read out by a change in photoacoustic signal frequency as an additional observable parameter for molecular photoacoustics. This molecular sensor mechanism could be generalized by genetically reprogramming other cell types to relocalize pigments via various mechanisms (Figure 2, lower right segment).

\section{TOWARD TRANSLATION TO CLINICAL PA NEUROIMAGING}

PA imaging has already found applications in thyroid imaging, breast imaging, dermatology, and sentinel lymph node detection (Steinberg et al., 2019). The main obstacle for neuroimaging with PA in humans is the skull, which constitutes a barrier to both excitation light and the emitted ultrasound.

Ex vivo PA imaging through the intact skin and skull was attempted in primates and visualized the superior sagittal sinus and prominent bridging veins (Yang and Wang, 2008). The same group also attempted imaging a phantom through a human skull and could at least detect major gyri from an excised canine brain (Nie et al., 2012).

An exciting opportunity, which has already been seized by US, is to image through the fontanelle in neonates' skull, which offers an acoustic window as the skull is not yet calcified. In clinical diagnosis, transfontanelle US imaging can be used to rule out hemorrhages, as well as hypoxic brain injuries (Dudink et al., 2020). Apart from these implementations in anatomical imaging, functional neonatal ultrasound in newborns proved useful for identifying ictal foci by tracking hemodynamic responses to massive brain activation during seizures (Demene et al., 2017).
Notwithstanding, MRI is still the preferred modality in the absence of an absolute contraindication, US being used mainly as a screening tool at the bedside and for disease follow-up.

Initial phantom experiments have been conducted to ascertain the feasibility of transfontanelle PA imaging (Wang et al., 2008). In a sheep model with a cranial window, combined imaging with US and PA could detect experimentally induced intraventricular and intraparenchymal hemorrhage (Hariri et al., 2017). It remains to be determined whether PA detection may substantially outperform the cheaper and more available standalone US systems in diagnostic accuracy for these pathologies.

PA may also play a role in the operating theater to identify structures that should not be lesioned or help determine the tumor-free resection margins. However, a technical challenge that needs to be overcome is to realize acoustic coupling of the brain to the US detector in a non-invasive and convenient way. In this context, recent contact-free realizations of PAM seek to detect the photoacoustic effect via a change in the refractory index, read out via a co-focused laser beam (Hajireza et al., 2017). This method for non-contact reflection mode PA sensing showed label-free image contrast similar to the established hematoxylin and eosin staining in a proof-of-principle study. There is ongoing research to apply this detection method also for imaging fresh brain samples and in situ implementations during surgery (Abbasi et al., 2019; Ecclestone et al., 2020). Significant challenges to implementing such an imaging strategy in vivo in the operation theater are represented by insufficient imaging depth at the low wavelengths ( $\sim 250 \mathrm{~nm})$.

Another possible implementation of intraoperative PA neuroimaging refers to the discrimination of peripheral nerves from the surrounding tissue. Recent phantom studies indicate superior contrast of peripheral nerves can be achieved with interventional PA/ultrasound devices as opposed to stand-alone US imaging system (Matthews et al., 2014; Mari et al., 2015), as also supported by in vivo experiments in mouse models (Li R. et al., 2016). Furthermore, diabetic polyneuropathy was found to cause significant increases in cross-sectional area in the median and sural nerves and decreased blood volume in PAI, which may indicate a possible advantage of combined PA/US over US alone (Yagihashi, 2018).

\section{CONCLUSIONS}

Rapid progress in laser technology and ultrasound detector arrays have allowed for a set of PAI systems, which address brain imaging at different temporal and spatial scales and effectively complement more established imaging techniques.

PA combines the advantages of mapping the biodistribution of photoabsorbers via multispectral illumination with the lower scattering of ultrasound to obtain informative resolution in tissues at depths beyond the reach of multiphoton detection.

In the rodent system, still probably the most frequently used model system in neuroscience, volumetric PA acquisition can also capture the whole brain but faster than MRI. However, in vivo differentiation of anatomical brain structures is currently not as detailed as in preclinical MRI, where cortical column mapping 
at $\sim 20$ micrometer isotropic resolution can be achieved (Wang et al., 2020).

Given that the rodent brain's geometry is quite similar between animals and much more static than, e.g., internal gastrointestinal tissue, anatomical resolution, and spectral unmixing can be rapidly and substantially improved by further refinement of reconstruction routines. This process can be supported by information on skull anatomy (Estrada et al., 2018), correction for photon flux, and correlation to complementary modalities (Attia et al., 2016; Gehrung et al., 2020), aided by machinelearning (Reiter and Lediju Bell, 2017; Davoudi et al., 2019).

Furthermore, PA sensitivity to hemoglobin absorption enables label-free quantification of brain oxygen metabolism in addition to fast detection of blood flow also accessible to ultrasound and SWIR imaging using blood-pool agents.

With ML-assisted unmixing algorithms, volumetric PA can deliver video-rate maps of oxygenation and blood volume from rodent brains (Olefir et al., 2020). Similar information can be inferred from MRI via modeling based on prescans and oxygenation challenges. These MRI data will be available at lower volume rates but already from humans. The native sensitivity of PA for hemodynamics is also proving to be beneficial to further investigate the physiology of neurovascular coupling via combined in vivo $\mathrm{PA} /$ multiphoton microscopes, which can improve the interpretation of macroscopic hemodynamic data. It is also conceivable to measure location-specific hemodynamic response functions (Lee et al., 2010) in multispectral PA by sequential electrical or optogenetic stimulation to improve hemodynamic neuroimaging fidelity by PA.

Concerning ex vivo PA imaging of the nervous system, a few tissue components such as neuromelanin, lipids, cytochrome, nucleic acids could be localized to provide more histological context before more complex fixation and staining protocols are carried out.

Notwithstanding these promising results, most whole-brain functional PA data has been acquired with custom-built setups, and the imaging equipment has yet to be made available to the broader neuroscience community.

A substantial increase in the value of PA imaging for preclinical neuroscience is also expected from the next generation of dynamic molecular sensors that will provide robust PA signals in response to brain activation events. There still seems to be a wide-open field for synthetic chemists to optimize NIR compounds for PA detection, which is an attractive application of these compounds given that the quantum yield in this wavelength range is already relatively low. However, in addition to optimal photophysical properties, equal attention must be paid to optimizing biodistribution. Solutions must be developed to target and deliver the compounds to the desired brain cells and into the relevant cellular compartments, such as the cytosol, while preserving their functionality. This subcellular targeting can be particularly challenging for synthetic nanostructures such as gold nanoparticles or nanotubes, although they may have superior extinction coefficients in the NIR range. Thus, innovative strategies for cellular targeting and delivery are needed that can successfully include semi-genetic approaches relying on mechanisms like enzymatic cleavage or intracellular aggregation.

Concerning genetically encoded contrast agents, further improvements to far-red shifted chromoproteins, that will ideally also be photoswitchable, will deliver more suitable candidates for molecular neuroimaging. Besides, there are ample opportunities to genetically control biosynthetic pigments as knowledge on multi-enzymatic biosynthetic pathways becomes available.

For behavioral neuroscientists, a manifest goal for mesoscopic, volumetric imaging techniques is to directly and non-invasively record and modulate neuronal activity in vivo. As discussed above, recent progress has been made in the development of reporters for molecular ultrasound (Maresca et al., 2018; Farhadi et al., 2019) and MRI. While these two modalities rely on wellcharacterized, mature technology that has already found its way into the neuroscience toolkit, the combination of fast volumetric PA imaging and robust sensors for neuronal activity would provide a potent platform for testing hypotheses on healthy and diseased brain function in small rodents.

Brain imaging with PA in humans is currently hampered by strong scattering and absorption due to the skull. Although technically feasible, PA imaging in infants would, of course, have to pass rigorous safety assessments and prove significantly superior to US diagnosis before being implemented in clinics. PA imaging of peripheral nerve lesions in the diagnosis of diabetic neuropathy may thus more easily find its way to the clinic. Furthermore, label-free PA microscopy could accelerate safe diagnosis of the margin of resection during brain surgery. There are currently intense efforts to miniaturize endoscopic PA imaging probes for gastrointestinal indications (Ali et al., 2021) that can likely be extended to lumbar puncture or needlebased biopsies. Image-assistance could thus be provided to these procedures, which may also be augmented by spectroscopy or FDA-approved imaging agents such as ICG.

The future development of PA imaging in neuroscience depends as much on the technological development and fabrication of robust brain imaging platforms as it does on the bioengineering of reliable reporters of brain activity. Thus, close collaboration between biomedical engineers, chemists, materials scientists, synthetic biologists, and data scientists is desirable to foster further innovations that will make PA neuroimaging an integral part of the laboratory and clinic.

\section{AUTHOR CONTRIBUTIONS}

All authors listed have made a substantial, direct and intellectual contribution to the work, and approved it for publication.

\section{ACKNOWLEDGMENTS}

We thank Dr. Felix Sigmund for his helpful feedback on the manuscript. 


\section{REFERENCES}

Abbasi, S., Le, M., Sonier, B., Dinakaran, D., Bigras, G., Bell, K., et al. (2019). Alloptical reflection-mode microscopic histology of unstained human tissues. Sci. Rep. 9:13392. doi: 10.1038/s41598-019-49849-9

Abbott, N. J., and Romero, I. A. (1996). Transporting therapeutics across the blood-brain barrier. Mol. Med. Today 2, 106-113. doi: 10.1016/1357-4310(96) 88720-X

Adam, Y., Kim, J. J., Lou, S., Zhao, Y., Xie, M. E., Brinks, D., et al. (2019). Voltage imaging and optogenetics reveal behaviour-dependent changes in hippocampal dynamics. Nature 569, 413-417. doi: 10.1038/s41586-019-1166-7

Aiello, M., Cavaliere, C., and Salvatore, M. (2016). Hybrid PET/MR imaging and brain connectivity. Front. Neurosci. 10:64. doi: 10.3389/fnins.2016.00064

Albers, F., Schmid, F., Wachsmuth, L., and Faber, C. (2018). Line scanning fMRI reveals earlier onset of optogenetically evoked BOLD response in rat somatosensory cortex as compared to sensory stimulation. Neuroimage 164, 144-154. doi: 10.1016/j.neuroimage.2016.12.059

Ali, F., and Kwan, A. C. (2019). Interpreting in vivo calcium signals from neuronal cell bodies, axons, and dendrites: a review. NPh 7:011402. doi: 10.1117/1.NPh. 7.1.011402

Ali, Z., Zakian, C., and Ntziachristos, V. (2021). Ultra-broadband axicon transducer for optoacoustic endoscopy. Sci. Rep. 11:1654. doi: 10.1038/s41598021-81117-7

Arellano, J. I., Benavides-Piccione, R., Defelipe, J., and Yuste, R. (2007). Ultrastructure of dendritic spines: correlation between synaptic and spine morphologies. Front. Neurosci. 1, 131-143. doi: 10.3389/neuro.01.1.1.010.2007

Attia, A. B. E., Ho, C. J. H., Chandrasekharan, P., Balasundaram, G., Tay, H. C., Burton, N. C., et al. (2016). Multispectral optoacoustic and MRI coregistration for molecular imaging of orthotopic model of human glioblastoma. J. Biophotonics 9, 701-708. doi: 10.1002/jbio.201500321

Balasundaram, G., Ding, L., Li, X., Attia, A. B. E., Dean-Ben, X. L., Ho, C. J. H., et al. (2018). Noninvasive anatomical and functional imaging of orthotopic glioblastoma development and therapy using multispectral optoacoustic tomography. Transl. Oncol. 11, 1251-1258. doi: 10.1016/j.tranon. 2018.07.001

Bando, Y., Sakamoto, M., Kim, S., Ayzenshtat, I., and Yuste, R. (2019). Comparative evaluation of genetically encoded voltage indicators. Cell Rep. 26, 802.e4813.e4. doi: 10.1016/j.celrep.2018.12.088

Bartlett, W. P., and Banker, G. A. (1984). An electron microscopic study of the development of axons and dendrites by hippocampal neurons in culture. I. Cells which develop without intercellular contacts. J. Neurosci. 4, 1944-1953. doi: 10.1523/JNEUROSCI.04-08-01944.1984

Bell, A. G. (1880). On the production and reproduction of sound by light. Am. J. Sci. 20, 305-324. doi: 10.2475/ajs.s3-20.118.305

Bell, K. L., Hajireza, P., Shi, W., and Zemp, R. J. (2017). Temporal evolution of lowcoherence reflectrometry signals in photoacoustic remote sensing microscopy. Appl. Opt. 56, 5172-5181. doi: 10.1364/AO.56.005172

Betzig, E., Patterson, G. H., Sougrat, R., Lindwasser, O. W., Olenych, S., Bonifacino, J. S., et al. (2006). Imaging intracellular fluorescent proteins at nanometer resolution. Science 313, 1642-1645. doi: 10.1126/science.1127344

Bruns, O. T., Bischof, T. S., Harris, D. K., Franke, D., Shi, Y., Riedemann, L., et al. (2017). Next-generation in vivo optical imaging with short-wave infrared quantum dots. Nat. Biomed. Eng. 1:56. doi: 10.1038/s41551-017-0056

Buehler, A., Herzog, E., Razansky, D., and Ntziachristos, V. (2010). Video rate optoacoustic tomography of mouse kidney perfusion. Opt. Lett. 35, 2475-2477. doi: 10.1364/OL.35.002475

Burton, N. C., Patel, M., Morscher, S., Driessen, W. H. P., Claussen, J., Beziere, N., et al. (2013). Multispectral opto-acoustic tomography (MSOT) of the brain and glioblastoma characterization. Neuroimage 65, 522-528. doi: 10.1016/j. neuroimage.2012.09.053

Byrd, B. K., Marois, M., Tichauer, K. M., Wirth, D. J., Hong, J., Leonor, J. P., et al. (2019). First experience imaging short-wave infrared fluorescence in a large animal: indocyanine green angiography of a pig brain. J. Biomed. Opt. 24, 1-4. doi: 10.1117/1.JBO.24.8.080501

Cai, X., Li, L., Krumholz, A., Guo, Z., Erpelding, T. N., Zhang, C., et al. (2012). Multi-scale molecular photoacoustic tomography of gene expression. PLoS One 7:e43999. doi: 10.1371/journal.pone.0043999
Cao, R., Li, J., Ning, B., Sun, N., Wang, T., Zuo, Z., et al. (2017). Functional and oxygen-metabolic photoacoustic microscopy of the awake mouse brain. Neuroimage 150, 77-87. doi: 10.1016/j.neuroimage.2017.01.049

Carr, J. A., Franke, D., Caram, J. R., Perkinson, C. F., Saif, M., Askoxylakis, V., et al. (2018). Shortwave infrared fluorescence imaging with the clinically approved near-infrared dye indocyanine green. Proc. Natl. Acad. Sci. U.S.A. 115, 4465-4470. doi: 10.1073/pnas.1718917115

Cetin, A., Komai, S., Eliava, M., Seeburg, P. H., and Osten, P. (2006). Stereotaxic gene delivery in the rodent brain. Nat. Protoc. 1, 3166-3173. doi: 10.1038/nprot. 2006.450

Challis, R. C., Ravindra Kumar, S., Chan, K. Y., Challis, C., Beadle, K., Jang, M. J., et al. (2019). Systemic AAV vectors for widespread and targeted gene delivery in rodents. Nat. Protoc. 14, 379-414. doi: 10.1038/s41596-018-0097-3

Chang, P.-C., Procissi, D., Bao, Q., Centeno, M. V., Baria, A., and Apkarian, A. V. (2016). Novel method for functional brain imaging in awake minimally restrained rats. J. Neurophysiol. 116, 61-80. doi: 10.1152/jn.01078.2015

Chavko, M., Nemoto, E. M., and Melick, J. A. (1993). Regional lipid composition in the rat brain. Mol. Chem. Neuropathol. 18, 123-131. doi: 10.1007/BF03160026

Chee, R. K. W., Li, Y., Zhang, W., Campbell, R. E., and Zemp, R. J. (2018). In vivo photoacoustic difference-spectra imaging of bacteria using photoswitchable chromoproteins. JBO 23:106006. doi: 10.1117/1.JBO.23.10.106006

Chen, F., Tillberg, P. W., and Boyden, E. S. (2015). Expansion microscopy. Science 347, 543-548. doi: 10.1126/science. 1260088

Chen, G., Wang, F., Dillenburger, B. C., Friedman, R. M., Chen, L. M., Gore, J. C., et al. (2012). Functional magnetic resonance imaging of awake monkeys: some approaches for improving imaging quality. Magn. Reson. Imaging 30, 36-47. doi: 10.1016/j.mri.2011.09.010

Chen, J., Liu, C., Hu, D., Wang, F., Wu, H., Gong, X., et al. (2016). Singlelayer MoS2 nanosheets with amplified photoacoustic effect for highly sensitive photoacoustic imaging of orthotopic brain tumors. Adv. Funct. Mater. 26, 8715-8725. doi: 10.1002/adfm.201603758

Chen, K.-T., Wei, K.-C., and Liu, H.-L. (2019). Theranostic strategy of focused ultrasound induced blood-brain barrier opening for CNS disease treatment. Front. Pharmacol. 10:86. doi: 10.3389/fphar.2019.00086

Chen, T.-W., Wardill, T. J., Sun, Y., Pulver, S. R., Renninger, S. L., Baohan, A., et al. (2013). Ultrasensitive fluorescent proteins for imaging neuronal activity. Nature 499, 295-300. doi: 10.1038/nature12354

Chen, X., Tong, C., Han, Z., Zhang, K., Bo, B., Feng, Y., et al. (2020). Sensory evoked fMRI paradigms in awake mice. Neuroimage 204:116242. doi: 10.1016/ j.neuroimage.2019.116242

Chen, Z., Deán-Ben, X. L., Gottschalk, S., and Razansky, D. (2017). Hybrid system for in vivo epifluorescence and $4 \mathrm{D}$ optoacoustic imaging. Opt. Lett. 42, 4577-4580. doi: 10.1364/OL.42.004577

Chung, K., and Deisseroth, K. (2013). CLARITY for mapping the nervous system. Nat. Methods 10, 508-513. doi: 10.1038/nmeth.2481

Cross, F. W., Al-Dhahir, R. K., Dyer, P. E., and MacRobert, A. J. (1987). Time-resolved photoacoustic studies of vascular tissue ablation at three laser wavelengths. Appl. Phys. Lett. 50, 1019-1021. doi: 10.1063/1.97994

Davoudi, N., Deán-Ben, X. L., and Razansky, D. (2019). Deep learning optoacoustic tomography with sparse data. Nat. Mach. Intellig. 1, 453-460. doi: 10.1038/ s42256-019-0095-3

de la Monte, S. M., and Tong, M. (2014). Brain metabolic dysfunction at the core of Alzheimer's disease. Biochem. Pharmacol. 88, 548-559. doi: 10.1016/j.bcp.2013. 12.012

Deán-Ben, X. L., Ford, S. J., and Razansky, D. (2015a). High-frame rate four dimensional optoacoustic tomography enables visualization of cardiovascular dynamics and mouse heart perfusion. Sci. Rep. 5:10133. doi: 10.1038/srep13240

Deán-Ben, X. L., Stiel, A. C., Jiang, Y., Ntziachristos, V., Westmeyer, G. G., and Razansky, D. (2015b). Light fluence normalization in turbid tissues via temporally unmixed multispectral optoacoustic tomography. Opt. Lett. 40, 4691-4694. doi: 10.1364/OL.40.004691

Deán-Ben, X. L., Gottschalk, S., Mc Larney, B., Shoham, S., and Razansky, D. (2017). Advanced optoacoustic methods for multiscale imaging of in vivo dynamics. Chem. Soc. Rev. 46, 2158-2198. doi: 10.1039/C6CS00765A

Deán-Ben, X. L., and Razansky, D. (2013). Portable spherical array probe for volumetric real-time optoacoustic imaging at centimeter-scale depths. Opt. Express 21, 28062-28071. doi: 10.1364/OE.21.028062 
Deán-Ben, X. L., Sela, G., Lauri, A., Kneipp, M., Ntziachristos, V., Westmeyer, G. G., et al. (2016). Functional optoacoustic neuro-tomography for scalable whole-brain monitoring of calcium indicators. Light Sci. Appl. 5:e16201. doi: 10.1038/lsa.2016.201

Deán-Ben, X. L., Weidenfeld, I., Degtyaruk, O., Ntziachristos, V., Stiel, A. C., and Razansky, D. (2020). Deep tissue volumetric optoacoustic tracking of individual circulating tumor cells in an intracardially perfused mouse model. Neoplasia 22, 441-446. doi: 10.1016/j.neo.2020.06.008

Deliolanis, N. C., Ale, A., Morscher, S., Burton, N. C., Schaefer, K., Radrich, K., et al. (2014). Deep-tissue reporter-gene imaging with fluorescence and optoacoustic tomography: a performance overview. Mol. Imaging Biol. 16, 652-660. doi: 10.1007/s11307-014-0728-1

Demene, C., Baranger, J., Bernal, M., Delanoe, C., Auvin, S., Biran, V., et al. (2017). Functional ultrasound imaging of brain activity in human newborns. Sci. Transl. Med. 9:eaah6756. doi: 10.1126/scitranslmed.aah6756

Devor, A., Sakadžiæ, S., Srinivasan, V. J., Yaseen, M. A., Nizar, K., Saisan, P. A., et al. (2012). Frontiers in optical imaging of cerebral blood flow and metabolism. J. Cereb. Blood Flow Metab. 32, 1259-1276. doi: 10.1038/jcbfm.2011.195

Dizeux, A., Gesnik, M., Ahnine, H., Blaize, K., Arcizet, F., Picaud, S., et al. (2019). Functional ultrasound imaging of the brain reveals propagation of task-related brain activity in behaving primates. Nat. Commun. 10, 1-9. doi: 10.1038/ s41467-019-09349-w

Dudink, J., Steggerda, S. J., and Horsch, S. (2020). State-of-the-art neonatal cerebral ultrasound: technique and reporting. Pediatr. Res. 87, 3-12. doi: 10.1038/ s41390-020-0776-y

Eberle, A. L., and Zeidler, D. (2018). Multi-beam scanning electron microscopy for high-throughput imaging in connectomics research. Front. Neuroanat. 12:112. doi: $10.3389 /$ fnana.2018.00112

Ecclestone, B. R., Bell, K., Abbasi, S., Dinakaran, D., van Landeghem, F. K. H., Mackey, J. R., et al. (2020). Improving maximal safe brain tumor resection with photoacoustic remote sensing microscopy. Sci. Rep. 10:17211. doi: 10.1038/ s41598-020-74160-3

Errico, C., Pierre, J., Pezet, S., Desailly, Y., Lenkei, Z., Couture, O., et al. (2015). Ultrafast ultrasound localization microscopy for deep super-resolution vascular imaging. Nature 527, 499-502. doi: 10.1038/nature16066

Esenaliev, R. O., Oraevsky, A. A., Jacques, S. L., and Tittel, F. K. (1996). "Laser opto-acoustic tomography for medical diagnostics: experiments with biological tissues," in Proceedings of the Biomedical Sensing, Imaging, and Tracking Technologies I (International Society for Optics and Photonics), San Jose, CA, 84-90. doi: 10.1117/12.238817

Estrada, H., Huang, X., Rebling, J., Zwack, M., Gottschalk, S., and Razansky, D. (2018). Virtual craniotomy for high-resolution optoacoustic brain microscopy. Sci. Rep. 8, 1-9. doi: 10.1038/s41598-017-18857-y

Fan, Q., Cheng, K., Hu, X., Ma, X., Zhang, R., Yang, M., et al. (2014). Transferring biomarker into molecular probe: melanin nanoparticle as a naturally active platform for multimodality imaging. J. Am. Chem. Soc. 136, 15185-15194. doi: 10.1021/ja505412p

Fan, Q., Cheng, K., Yang, Z., Zhang, R., Yang, M., Hu, X., et al. (2015). Perylenediimide-based nanoparticles as highly efficient photoacoustic agents for deep brain tumor imaging in living mice. Adv. Mater. 27, 843-847. doi: 10.1002/ adma.201402972

Farhadi, A., Ho, G. H., Sawyer, D. P., Bourdeau, R. W., and Shapiro, M. G. (2019). Ultrasound imaging of gene expression in mammalian cells. Science 365, 1469-1475. doi: 10.1126/science.aax4804

Filonov, G. S., Krumholz, A., Xia, J., Yao, J., Wang, L. V., and Verkhusha, V. V. (2012). Deep-tissue photoacoustic tomography of a genetically encoded nearinfrared fluorescent probe. Angew. Chem. Int. Ed Engl. 51, 1448-1451. doi: 10.1002/anie.201107026

Foust, K. D., Nurre, E., Montgomery, C. L., Hernandez, A., Chan, C. M., and Kaspar, B. K. (2009). Intravascular AAV9 preferentially targets neonatal neurons and adult astrocytes. Nat. Biotechnol. 27, 59-65. doi: 10.1038/nbt.1515

Friston, K. J. (1994). Functional and effective connectivity in neuroimaging: a synthesis. Hum. Brain Mapp. 2, 56-78. doi: 10.1002/hbm.460020107

Galanzha, E. I., Viegas, M. G., Malinsky, T. I., Melerzanov, A. V., Juratli, M. A., Sarimollaoglu, M., et al. (2016). In vivo acoustic and photoacoustic focusing of circulating cells. Sci. Rep. 6:21531. doi: 10.1038/srep21531

Gauthier, C. J., and Fan, A. P. (2019). BOLD signal physiology: models and applications. Neuroimage 187, 116-127. doi: 10.1016/j.neuroimage.2018.03.018
Gehrung, M., Tomaszewski, M., McIntyre, D., Disselhorst, J., and Bohndiek, S. (2020). Co-registration of optoacoustic tomography and magnetic resonance imaging data from murine tumour models. Photoacoustics 18:100147. doi: 10.1016/j.pacs.2019.100147

Gipson, C. D., and Olive, M. F. (2017). Structural and functional plasticity of dendritic spines - root or result of behavior? Genes Brain Behav. 16, 101-117. doi: $10.1111 / \mathrm{gbb} .12324$

Gottschalk, S., Degtyaruk, O., Mc Larney, B., Rebling, J., Deán-Ben, X. L., Shoham, S., et al. (2019a). Isolated murine brain model for large-scale optoacoustic calcium imaging. Front. Neurosci. 13:290. doi: 10.3389/fnins.2019.00290

Gottschalk, S., Degtyaruk, O., Mc Larney, B., Rebling, J., Hutter, M. A., Deán-Ben, X. L., et al. (2019b). Rapid volumetric optoacoustic imaging of neural dynamics across the mouse brain. Nat. Biomed. Eng. 3, 392-401. doi: 10.1038/s41551019-0372-9

Gottschalk, S., Fehm, T. F., Deán-Ben, X. L., Tsytsarev, V., and Razanksy, D. (2017). Concurrent electrophysiology and optoacoustic tomography enable visualization of deep thalamocortical activity in an acute mouse model of focal epilepsy. Opt. Life Sci. Cong. 6:BrW3B. doi: 10.1364/BRAIN.2017. BrW3B.6

Grinvald, A., and Hildesheim, R. (2004). VSDI: a new era in functional imaging of cortical dynamics. Nat. Rev. Neurosci. 5, 874-885. doi: 10.1038/nrn1536

Grinvald, A., Ross, W. N., and Farber, I. (1981). Simultaneous optical measurements of electrical activity from multiple sites on processes of cultured neurons. Proc. Natl. Acad. Sci. U.S.A. 78, 3245-3249. doi: 10.1073/pnas.78.5. 3245

Guo, B., Sheng, Z., Hu, D., Liu, C., Zheng, H., and Liu, B. (2018). Through scalp and skull NIR-II photothermal therapy of deep orthotopic brain tumors with precise photoacoustic imaging guidance. Adv. Mater. 30:e1802591. doi: 10.1002/adma. 201802591

Gurevich, V. V., and Gurevich, E. V. (2019). GPCR signaling regulation: the role of GRKs and arrestins. Front. Pharmacol. 10:125. doi: 10.3389/fphar.2019.00125

Haggerty, D. L., Grecco, G. G., Reeves, K. C., and Atwood, B. (2020). Adenoassociated viral vectors in neuroscience research. Mol. Ther. Methods Clin. Dev. 17, 69-82. doi: 10.1016/j.omtm.2019.11.012

Hai, P., Qu, Y., Li, Y., Zhu, L., Shmuylovich, L., Cornelius, L. A., et al. (2020). Label-free high-throughput photoacoustic tomography of suspected circulating melanoma tumor cells in patients in vivo. J. Biomed. Opt. 25, 1-17. doi: 10. 1117/1.JBO.25.3.036002

Hajireza, P., Shi, W., Bell, K., Paproski, R. J., and Zemp, R. J. (2017). Noninterferometric photoacoustic remote sensing microscopy. Light Sci. Appl. 6:e16278. doi: 10.1038/lsa.2016.278

Hariri, A., Tavakoli, E., Adabi, S., Gelovani, J., and Avanaki, M. R. N. (2017). "Functional photoacoustic tomography for neonatal brain imaging: developments and challenges," in Proceedings of the Photons Plus Ultrasound: Imaging and Sensing 2017 (International Society for Optics and Photonics), San Jose, CA. doi: 10.1117/12.2254861

Harris, S., Ma, H., Zhao, M., Boorman, L., Zheng, Y., Kennerley, A., et al. (2014). Coupling between gamma-band power and cerebral blood volume during recurrent acute neocortical seizures. Neuroimage 97, 62-70. doi: 10.1016/j. neuroimage.2014.04.014

Hildebrand, D. G. C., Cicconet, M., Torres, R. M., Choi, W., Quan, T. M., Moon, J., et al. (2017). Whole-brain serial-section electron microscopy in larval zebrafish. Nature 545, 345-349. doi: 10.1038/nature22356

Hoang, H., Sato, M.-A., Shinomoto, S., Tsutsumi, S., Hashizume, M., Ishikawa, T., et al. (2020). Improved hyperacuity estimation of spike timing from calcium imaging. Sci. Rep. 10:17844. doi: 10.1038/s41598-020-74672-y

Hoelen, C. G. A., de Mul, F. F. M., Pongers, R., and Dekker, A. (1998). Threedimensional photoacoustic imaging of blood vessels in tissue. Opt. Lett. OL 23, 648-650. doi: 10.1364/OL.23.000648

Hofmann, U. A. T., Fabritius, A., Rebling, J., Estrada, H., Deán-Ben, X. L., Griesbeck, O., et al. (2019). High-throughput platform for optoacoustic probing of genetically encoded calcium ion indicators. iScience 22, 400-408. doi: 10 . 1016/j.isci.2019.11.034

Hosseinaee, Z., Le, M., Bell, K., and Reza, P. H. (2020). Towards non-contact photoacoustic imaging [review]. Photoacoustics 20:100207. doi: 10.1016/j.pacs. 2020.100207

Houston, Z. H., Bunt, J., Chen, K.-S., Puttick, S., Howard, C. B., Fletcher, N. L., et al. (2020). Understanding the uptake of nanomedicines at different stages 
of brain cancer using a modular nanocarrier platform and precision bispecific antibodies. ACS Cent. Sci. 6, 727-738. doi: 10.1021/acscentsci.9b01299

Hsu, H.-C., Li, L., Yao, J., Wong, T. T. W., Shi, J., Chen, R., et al. (2018). Dual-axis illumination for virtually augmenting the detection view of optical-resolution photoacoustic microscopy. JBO 23:076001. doi: 10.1117/1.JBO.23.7.076001

Hu, S., Gonzales, E., Soetikno, B., Gong, E., Yan, P., Maslov, K., et al. (2011a). "Optical-resolution photoacoustic microscopy of ischemic stroke," in Proceedings of the Photons Plus Ultrasound: Imaging and Sensing 2011 (International Society for Optics and Photonics), San Jose, CA. doi: 10.1117/12. 874366

Hu, S., Maslov, K., and Wang, L. V. (2011b). Second-generation optical-resolution photoacoustic microscopy with improved sensitivity and speed. Opt. Lett. 36, 1134-1136. doi: 10.1364/OL.36.001134

Hu, S., and Wang, L. V. (2013). Optical-resolution photoacoustic microscopy: auscultation of biological systems at the cellular level. Biophys. J. 105, 841-847. doi: 10.1016/j.bpj.2013.07.017

Hu, S., Yan, P., Maslov, K., Lee, J.-M., and Wang, L. V. (2009). Intravital imaging of amyloid plaques in a transgenic mouse model using optical-resolution photoacoustic microscopy. Opt. Lett. 34, 3899-3901. doi: 10.1364/OL.34. 003899

Huneau, C., Benali, H., and Chabriat, H. (2015). Investigating human neurovascular coupling using functional neuroimaging: a critical review of dynamic models. Front. Neurosci. 9:467. doi: 10.3389/fnins.2015.00467

Imbault, M., Chauvet, D., Gennisson, J.-L., Capelle, L., and Tanter, M. (2017). Intraoperative functional ultrasound imaging of human brain activity. Sci. Rep. 7:7304. doi: 10.1038/s41598-017-06474-8

Ingusci, S., Verlengia, G., Soukupova, M., Zucchini, S., and Simonato, M. (2019). Gene therapy tools for brain diseases. Front. Pharmacol. 10:724. doi: 10.3389/ fphar.2019.00724

Jbabdi, S., Sotiropoulos, S. N., Haber, S. N., Van Essen, D. C., and Behrens, T. E. (2015). Measuring macroscopic brain connections in vivo. Nat. Neurosci. 18, 1546-1555. doi: 10.1038/nn.4134

Jeon, S., Kim, J., Lee, D., Baik, J. W., and Kim, C. (2019). Review on practical photoacoustic microscopy. Photoacoustics 15:100141. doi: 10.1016/j.pacs.2019. 100141

Kang, J., Zhang, H. K., Kadam, S. D., Fedorko, J., Valentine, H., Malla, A. P., et al. (2019). Transcranial recording of electrophysiological neural activity in the rodent brain in vivo using functional photoacoustic imaging of nearinfrared voltage-sensitive dye. Front. Neurosci. 13:579. doi: 10.3389/fnins.2019. 00579

Kang, N.-Y., Park, S.-J., Ang, X. W. E., Samanta, A., Driessen, W. H. P., Ntziachristos, V., et al. (2014). A macrophage uptaking near-infrared chemical probe CDnir7 for in vivo imaging of inflammation. Chem. Commun. 50, 6589-6591. doi: 10.1039/c4cc02038c

Kim, J., Kim, J. Y., Jeon, S., Baik, J. W., Cho, S. H., and Kim, C. (2019). Superresolution localization photoacoustic microscopy using intrinsic red blood cells as contrast absorbers. Light Sci. Appl. 8:103. doi: 10.1038/s41377-019-0220-4

Kircher, M. F., de la Zerda, A., Jokerst, J. V., Zavaleta, C. L., Kempen, P. J., Mittra, E., et al. (2012). A brain tumor molecular imaging strategy using a new triplemodality MRI-photoacoustic-Raman nanoparticle. Nat. Med. 18, 829-834. doi: $10.1038 / \mathrm{nm} .2721$

Kneipp, M., Turner, J., Hambauer, S., Krieg, S. M., Lehmberg, J., Lindauer, U., et al. (2014). Functional real-time optoacoustic imaging of middle cerebral artery occlusion in mice. PLoS One 9:e96118. doi: 10.1371/journal.pone. 0096118

Köker, T., Tang, N., Tian, C., Zhang, W., Wang, X., Martel, R., et al. (2018). Cellular imaging by targeted assembly of hot-spot SERS and photoacoustic nanoprobes using split-fluorescent protein scaffolds. Nat. Commun. 9:607. doi: 10.1038/s41467-018-03046-w

Kong, L., Tang, J., Little, J. P., Yu, Y., Lämmermann, T., Lin, C. P., et al. (2015). Continuous volumetric imaging via an optical phase-locked ultrasound lens. Nat. Methods 12, 759-762. doi: 10.1038/nmeth.3476

Kruger, R. A., Liu, P., Fang, Y. R., and Appledorn, C. R. (1995). Photoacoustic ultrasound (PAUS)-reconstruction tomography. Med. Phys. 22, 1605-1609. doi: $10.1118 / 1.597429$

Krumholz, A., Vanvickle-Chavez, S. J., Yao, J., Fleming, T. P., Gillanders, W. E., and Wang, L. V. (2011). Photoacoustic microscopy of tyrosinase reporter gene in vivo. J. Biomed. Opt. 16:080503. doi: 10.1117/1.3606568
Kwan, A. C., and Dan, Y. (2012). Dissection of cortical microcircuits by singleneuron stimulation in vivo. Curr. Biol. 22, 1459-1467. doi: 10.1016/j.cub.2012. 06.007

Lake, E. M. R., Ge, X., Shen, X., Herman, P., Hyder, F., Cardin, J. A., et al. (2020). Simultaneous cortex-wide fluorescence $\mathrm{Ca} 2+$ imaging and whole-brain fMRI. Nat. Methods 17, 1262-1271. doi: 10.1038/s41592-020-00984-6

Lauri, A., Soliman, D., Omar, M., Stelzl, A., Ntziachristos, V., and Westmeyer, G. G. (2019). Whole-cell photoacoustic sensor based on pigment relocalization. ACS Sens. 4, 603-612. doi: 10.1021/acssensors.8b01319

Lee, J. H., Durand, R., Gradinaru, V., Zhang, F., Goshen, I., Kim, D.-S., et al. (2010). Global and local fMRI signals driven by neurons defined optogenetically by type and wiring. Nature 465, 788-792. doi: 10.1038/nature09108

Lee, S., Li, X., Lee, D., Yoon, J., and Kim, C. (2018). "Photoacoustic imaging of tumor targeting with biotin conjugated nanostructured phthalocyanine assemblies," in Proceedings of the Photons Plus Ultrasound: Imaging and Sensing 2018 (International Society for Optics and Photonics), San Jose, CA. doi: 10.1117/ 12.2292784

Li, L., Xia, J., Li, G., Garcia-Uribe, A., Sheng, Q., Anastasio, M. A., et al. (2016). Label-free photoacoustic tomography of whole mouse brain structures ex vivo. Neurophotonics 3:035001. doi: 10.1117/1.NPh.3.3.035001

Li, L., Zemp, R. J., Lungu, G., Stoica, G., and Wang, L. V. (2007). Photoacoustic imaging of lacZ gene expression in vivo. J. Biomed. Opt. 12:020504. doi: 10. $1117 / 1.2717531$

Li, L., Zhang, H. F., Zemp, R. J., Maslov, K., and Wang, L. (2008). Simultaneous imaging of a lacZ-marked tumor and microvasculature morphology in vivo by dual-wavelength photoacoustic microscopy. J. Innov. Opt. Health Sci. 1, 207-215. doi: 10.1142/S1793545808000212

Li, M., Oh, J., Xie, X., Ku, G., Wang, W., Li, C., et al. (2008). Simultaneous molecular and hypoxia imaging of brain tumors in vivo using spectroscopic photoacoustic tomography. Proc. IEEE 96, 481-489. doi: 10.1109/JPROC.2007. 913515

Li, M.-L., Zhang, H. E., Maslov, K., Stoica, G., and Wang, L. V. (2006). Improved in vivo photoacoustic microscopy based on a virtual-detector concept. Opt. Lett. 31, 474-476. doi: 10.1364/OL.31.000474

Li, R., Phillips, E., Wang, P., Goergen, C. J., and Cheng, J.-X. (2016). Labelfree in vivo imaging of peripheral nerve by multispectral photoacoustic tomography. J. Biophotonics 9, 124-128. doi: 10.1002/jbio.201500004

Li, W., Chen, R., Lv, J., Wang, H., Liu, Y., Peng, Y., et al. (2018). In vivo photoacoustic imaging of brain injury and rehabilitation by high-efficient nearinfrared dye labeled mesenchymal stem cells with enhanced brain barrier permeability. Adv. Sci. 5:1700277. doi: 10.1002/advs.201700277

Li, Y., Forbrich, A., Wu, J., Shao, P., Campbell, R. E., and Zemp, R. (2016). Engineering dark chromoprotein reporters for photoacoustic microscopy and FRET imaging. Sci. Rep. 6:22129. doi: 10.1038/srep22129

Liao, L.-D., Li, M.-L., Lai, H.-Y., Chen, Y.-Y., and Thakor, N. V. (2013). Study of neurovascular coupling functions for transient focal cerebral ischemia in rats using electrocorticography functional photoacoustic microscopy (ECoGfPAM). Conf. Proc. IEEE Eng. Med. Biol. Soc. 2013, 1799-1802.

Liao, L.-D., Li, M.-L., Lai, H.-Y., Shih, Y.-Y. I., Lo, Y.-C., Tsang, S., et al. (2010). Imaging brain hemodynamic changes during rat forepaw electrical stimulation using functional photoacoustic microscopy. Neuroimage 52, 562-570. doi: 10.1016/j.neuroimage.2010.03.065

Liao, L.-D., Lin, C.-T., Shih, Y.-Y. I., Duong, T. Q., Lai, H.-Y., Wang, P.-H., et al. (2012). Transcranial imaging of functional cerebral hemodynamic changes in single blood vessels using in vivo photoacoustic microscopy. J. Cereb. Blood Flow Metab. 32, 938-951. doi: 10.1038/jcbfm.2012.42

Lin, L., Xia, J., Wong, T. T. W., Zhang, R., and Wang, L. V. (2015). "In vivo deep brain imaging of rats using oral-cavity illuminated photoacoustic computed tomography," in Proceedings of the Photons Plus Ultrasound: Imaging and Sensing 2015 (International Society for Optics and Photonics), San Jose, CA. doi: $10.1117 / 12.2076482$

Liu, L., Chen, Q., Wen, L., Li, C., Qin, H., and Xing, D. (2019). Photoacoustic therapy for precise eradication of glioblastoma with a tumor site blood-brain barrier permeability upregulating nanoparticle. Adv. Funct. Mater. 29:1808601. doi: $10.1002 /$ adfm.201904827

Livet, J., Weissman, T. A., Kang, H., Draft, R. W., Lu, J., Bennis, R. A., et al. (2007). Transgenic strategies for combinatorial expression of fluorescent proteins in the nervous system. Nature 450, 56-62. doi: 10.1038/nature06293 
Logothetis, N. K. (2008). What we can do and what we cannot do with fMRI. Nature 453, 869-878. doi: 10.1038/nature06976

Luís Deán-Ben, X., and Razansky, D. (2014). Adding fifth dimension to optoacoustic imaging: volumetric time-resolved spectrally enriched tomography. Light Sci. Appl. 3:e137. doi: 10.1038/lsa.2014.18

Luís Dean-Ben, X., and Razansky, D. (2018). Localization optoacoustic tomography. Light Sci. Appl. 7:18004. doi: 10.1038/lsa.2018.4

Luke, G. P., Myers, J. N., Emelianov, S. Y., and Sokolov, K. V. (2014). Sentinel lymph node biopsy revisited: ultrasound-guided photoacoustic detection of micrometastases using molecularly targeted plasmonic nanosensors. Cancer Res. 74, 5397-5408. doi: 10.1158/0008-5472.CAN-14-0796

Lutzweiler, C., and Razansky, D. (2013). Optoacoustic imaging and tomography: reconstruction approaches and outstanding challenges in image performance and quantification. Sensors 13, 7345-7384. doi: 10.3390/s130607345

Lv, J., Li, S., Zhang, J., Duan, F., Wu, Z., Chen, R., et al. (2020). In vivo photoacoustic imaging dynamically monitors the structural and functional changes of ischemic stroke at a very early stage. Theranostics 10, 816-828. doi: $10.7150 /$ thno. 38554

Macé, E., Montaldo, G., Cohen, I., Baulac, M., Fink, M., and Tanter, M. (2011). Functional ultrasound imaging of the brain. Nat. Methods 8, 662-664. doi: 10.1038/nmeth.1641

Magistretti, P. J., and Pellerin, L. (1999). Cellular mechanisms of brain energy metabolism and their relevance to functional brain imaging. Philos. Trans. $R$. Soc. Lond. B Biol. Sci. 354, 1155-1163. doi: 10.1098/rstb.1999.0471

Maknojia, S., Churchill, N. W., Schweizer, T. A., and Graham, S. J. (2019). Resting state fMRI: going through the motions. Front. Neurosci. 13:825. doi: 10.3389/ fnins.2019.00825

Malvaut, S., Constantinescu, V.-S., Dehez, H., Doric, S., and Saghatelyan, A. (2020). Deciphering brain function by miniaturized fluorescence microscopy in freely behaving animals. Front. Neurosci. 14:819. doi: 10.3389/fnins.2020.00819

Manfredsson, F. P., Rising, A. C., and Mandel, R. J. (2009). AAV9: a potential blood-brain barrier buster. Mol. Ther. 17, 403-405. doi: 10.1038/mt.2009.15

Manivasagan, P., Quang Bui, N., Bharathiraja, S., Santha Moorthy, M., Oh, Y.-O., Song, K., et al. (2017). Multifunctional biocompatible chitosanpolypyrrole nanocomposites as novel agents for photoacoustic imagingguided photothermal ablation of cancer. Sci. Rep. 7:43593. doi: 10.1038/srep 43593

Mank, M., Santos, A. F., Direnberger, S., Mrsic-Flogel, T. D., Hofer, S. B., Stein, V., et al. (2008). A genetically encoded calcium indicator for chronic in vivo two-photon imaging. Nat. Methods 5, 805-811. doi: 10.1038/nmeth.1243

Mano, T., Albanese, A., Dodt, H.-U., Erturk, A., Gradinaru, V., Treweek, J. B., et al. (2018). Whole-brain analysis of cells and circuits by tissue clearing and lightsheet microscopy. J. Neurosci. 38, 9330-9337. doi: 10.1523/JNEUROSCI.167718.2018

Manohar, S., and Razansky, D. (2016). Photoacoustics: a historical review. Adv. Opt. Photon. AOP 8, 586-617. doi: 10.1364/AOP.8.000586

Maresca, D., Lakshmanan, A., Abedi, M., Bar-Zion, A., Farhadi, A., Lu, G. J., et al. (2018). Biomolecular ultrasound and sonogenetics. Annu. Rev. Chem. Biomol. Eng. 9, 229-252. doi: 10.1146/annurev-chembioeng-060817-084034

Mari, J. M., Xia, W., West, S. J., and Desjardins, A. E. (2015). Interventional multispectral photoacoustic imaging with a clinical ultrasound probe for discriminating nerves and tendons: an ex vivo pilot study. J. Biomed. Opt. 20:110503. doi: 10.1117/1.JBO.20.11.110503

Märk, J., Dortay, H., Wagener, A., Zhang, E., Buchmann, J., Grötzinger, C., et al. (2018). Dual-wavelength 3D photoacoustic imaging of mammalian cells using a photoswitchable phytochrome reporter protein. Commun. Phys. 1:3. doi: 10.1038/s42005-017-0003-2

Marriott, G., Mao, S., Sakata, T., Ran, J., Jackson, D. K., Petchprayoon, C., et al. (2008). Optical lock-in detection imaging microscopy for contrast-enhanced imaging in living cells. Proc. Natl. Acad. Sci. U.S.A. 105, 17789-17794. doi: 10.1073/pnas.0808882105

Maslov, K., Stoica, G., and Wang, L. V. (2005). In vivo dark-field reflection-mode photoacoustic microscopy. Opt. Lett. 30, 625-627. doi: 10.1364/OL.30.000625

Maslov, K., Zhang, H. F., Hu, S., and Wang, L. V. (2008). Optical-resolution photoacoustic microscopy for in vivo imaging of single capillaries. Opt. Lett. OL 33, 929-931. doi: 10.1364/OL.33.000929

Masujima, T., Munekane, Y., Kawai, C., Yoshida, H., Imai, H., Juing-Yi, L., et al. (1988). "Photoacoustic Imaging Immunoassay for Biological Component
Microanalysis," in Photoacoustic and Photothermal Phenomena, eds J. Pelzl and P. Hess (Berlin: Springer), 558-560. doi: 10.1007/978-3-540-48181-2_152

Matthews, T. P., Zhang, C., Yao, D.-K., Maslov, K., and Wang, L. V. (2014). Labelfree photoacoustic microscopy of peripheral nerves. J. Biomed. Opt. 19:16004. doi: 10.1117/1.JBO.19.1.016004

Mc Larney, B., Hutter, M. A., Degtyaruk, O., Deán-Ben, X. L., and Razansky, D. (2020). Monitoring of stimulus evoked murine somatosensory cortex hemodynamic activity with volumetric multi-spectral optoacoustic tomography. Front. Neurosci. 14:536. doi: 10.3389/fnins.2020.00536

McGraw, C. M., Ward, C. S., and Samaco, R. C. (2017). Genetic rodent models of brain disorders: Perspectives on experimental approaches and therapeutic strategies. Am. J. Med. Genet. C Semin. Med. Genet. 175, 368-379. doi: 10.1002/ ajmg.c. 31570

Milej, D., Abdalmalak, A., Desjardins, L., Ahmed, H., Lee, T.-Y., Diop, M., et al. (2017). Quantification of blood-brain barrier permeability by dynamic contrastenhanced NIRS. Sci. Rep. 7, 1-8. doi: 10.1038/s41598-017-01922-x

Mishra, K., Stankevych, M., Fuenzalida-Werner, J. P., Grassmann, S., Gujrati, V., Huang, Y., (2020). Multiplexed whole-animal imaging with reversibly switchable optoacoustic proteins. Sci. Adv. 6:eaaz6293. doi: 10.1126/sciadv. aaz6293

Miyawaki, A., Llopis, J., Heim, R., McCaffery, J. M., Adams, J. A., Ikura, M., et al. (1997). Fluorescent indicators for $\mathrm{Ca} 2+$ based on green fluorescent proteins and calmodulin. Nature 388, 882-887. doi: 10.1038/42264

Moeller, F., Siniatchkin, M., and Gotman, J. (2020). "Simultaneous EEG and fMRI recordings (EEG-fMRI)," in fMRI: Basics and Clinical Applications, eds S. Ulmer and O. Jansen (Cham: Springer), 175-191. doi: 10.1007/978-3-030-41874-8_13

Motta, A., Berning, M., Boergens, K. M., Staffler, B., Beining, M., Loomba, S., et al. (2019). Dense connectomic reconstruction in layer 4 of the somatosensory cortex. Science 366:eaay3134. doi: 10.1126/science.aay3134

Muller, A., Joseph, V., Slesinger, P. A., and Kleinfeld, D. (2014). Cell-based reporters reveal in vivo dynamics of dopamine and norepinephrine release in murine cortex. Nat. Methods 11, 1245-1252. doi: 10.1038/nmeth.3151

Muñoz, M. F., Puebla, M., and Figueroa, X. F. (2015). Control of the neurovascular coupling by nitric oxide-dependent regulation of astrocytic $\mathrm{Ca} 2+$ signaling. Front. Cell. Neurosci. 9:59. doi: 10.3389/fncel.2015.00059

Nel, A., Ruoslahti, E., and Meng, H. (2017). New insights into "permeability" as in the enhanced permeability and retention effect of cancer nanotherapeutics. ACS Nano 11, 9567-9569. doi: 10.1021/acsnano.7b07214

Neuschmelting, V., Harmsen, S., Beziere, N., Lockau, H., Hsu, H.-T., Huang, R., et al. (2018). Dual-modality surface-enhanced resonance raman scattering and multispectral optoacoustic tomography nanoparticle approach for brain tumor delineation. Small 14:e1800740. doi: 10.1002/smll.201800740

Ni, R., Rudin, M., and Klohs, J. (2018). Cortical hypoperfusion and reduced cerebral metabolic rate of oxygen in the $\operatorname{arcA} \beta$ mouse model of Alzheimer's disease. Photoacoustics 10, 38-47. doi: 10.1016/j.pacs.2018.04.001

Nie, L., Cai, X., Maslov, K., Garcia-Uribe, A., Anastasio, M. A., and Wang, L. V. (2012). Photoacoustic tomography through a whole adult human skull with a photon recycler. J. Biomed. Opt. 17:110506. doi: 10.1117/1.JBO.17.11.110506

Ntziachristos, V. (2010). Going deeper than microscopy: the optical imaging frontier in biology. Nat. Methods 7, 603-614. doi: 10.1038/nmeth.1483

Ntziachristos, V., and Razansky, D. (2010). Molecular imaging by means of multispectral optoacoustic tomography (MSOT). Chem. Rev. 110, 2783-2794. doi: $10.1021 /$ cr9002566

O'Brien, J. A., and Lummis, S. C. R. (2006). Biolistic transfection of neuronal cultures using a hand-held gene gun. Nat. Protoc. 1, 977-981. doi: 10.1038/ nprot.2006.145

Ogawa, S., Lee, T. M., Kay, A. R., and Tank, D. W. (1990). Brain magnetic resonance imaging with contrast dependent on blood oxygenation. Proc. Natl. Acad. Sci. U.S.A. $87,9868-9872$. doi: $10.1073 /$ pnas.87.24.9868

Ogawa, S., Tank, D. W., Menon, R., Ellermann, J. M., Kim, S. G., Merkle, H., et al. (1992). Intrinsic signal changes accompanying sensory stimulation: functional brain mapping with magnetic resonance imaging. Proc. Natl. Acad. Sci. U.S.A. 89, 5951-5955. doi: 10.1073/pnas.89.13.5951

Olefir, I., Ghazaryan, A., Yang, H., Malekzadeh-Najafabadi, J., Glasl, S., Symvoulidis, P., et al. (2019). Spatial and spectral mapping and decomposition of neural dynamics and organization of the mouse brain with multispectral optoacoustic tomography. Cell Rep. 26, 2833.e12-2846.e12. doi: 10.1016/j. celrep.2019.02.020 
Olefir, I., Mercep, E., Burton, N. C., Ovsepian, S. V., and Ntziachristos, V. (2016). Hybrid multispectral optoacoustic and ultrasound tomography for morphological and physiological brain imaging. J. Biomed. Opt. 21:86005. doi: 10.1117/1.JBO.21.8.086005

Olefir, I., Tzoumas, S., Restivo, C., Mohajerani, P., Xing, L., and Ntziachristos, V. (2020). Deep learning-based spectral unmixing for optoacoustic imaging of tissue oxygen saturation. IEEE Trans. Med. Imaging 39, 3643-3654. doi: 10.1109/TMI.2020.3001750

Omar, M., Soliman, D., Gateau, J., and Ntziachristos, V. (2014). Ultrawideband reflection-mode optoacoustic mesoscopy. Opt. Lett. 39, 3911-3914. doi: 10. 1364/OL.39.003911

Oraevsky, A. A., Esenaliev, R. O., Jacques, S. L., and Tittel, F. K. (1996). "Laser optic-acoustic tomography for medical diagnostics: principles," in Proceedings of the Biomedical Sensing, Imaging, and Tracking Technologies I (International Society for Optics and Photonics), San Jose, CA, 22-31. doi: 10.1117/12.238786

Oraevsky, A. A., Jacques, S. L., and Tittel, F. K. (1993). "Determination of tissue optical properties by piezoelectric detection of laser-induced stress waves," in Proceedings of the Laser-Tissue Interaction IV (International Society for Optics and Photonics), San Jose, CA, 86-101. doi: 10.1117/12.147694

Oraevsky, A. A., Jacques, S. L., and Tittel, F. K. (1997). Measurement of tissue optical properties by time-resolved detection of laser-induced transient stress. Appl. Opt. AO 36, 402-415. doi: 10.1364/AO.36.000402

Ovsepian, S. V., Olefir, I., Westmeyer, G., Razansky, D., and Ntziachristos, V. (2017). Pushing the boundaries of neuroimaging with optoacoustics. Neuron 96, 966-988. doi: 10.1016/j.neuron.2017.10.022

Pachitariu, M., Stringer, C., and Harris, K. D. (2018). Robustness of spike deconvolution for neuronal calcium imaging. J. Neurosci. 38, 7976-7985. doi: 10.1523/JNEUROSCI.3339-17.2018

Panzera, L. C., and Hoppa, M. B. (2019). Genetically encoded voltage indicators are illuminating subcellular physiology of the axon. Front. Cell. Neurosci. 13:52. doi: 10.3389/fncel.2019.00052

Paproski, R. J., Heinmiller, A., Wachowicz, K., and Zemp, R. J. (2014). Multiwavelength photoacoustic imaging of inducible tyrosinase reporter gene expression in xenograft tumors. Sci. Rep. 4:5329. doi: 10.1038/srep05329

Pardridge, W. M. (2005). The blood-brain barrier: bottleneck in brain drug development. NeuroRx 2, 3-14. doi: 10.1602/neurorx.2.1.3

Park, S., Lee, C., Kim, J., and Kim, C. (2014). Acoustic resolution photoacoustic microscopy. Biomed. Eng. Lett. 4, 213-222. doi: 10.1007/s13534-014-0153-z

Park, S.-J., Ho, C. J. H., Arai, S., Samanta, A., Olivo, M., and Chang, Y.-T. (2019). Visualizing Alzheimer's disease mouse brain with multispectral optoacoustic tomography using a fluorescent probe, CDnir7. Sci. Rep. 9:12052. doi: 10.1038/ s41598-019-48329-4

Patriarchi, T., Cho, J. R., Merten, K., Howe, M. W., Marley, A., Xiong, W.-H., et al. (2018). Ultrafast neuronal imaging of dopamine dynamics with designed genetically encoded sensors. Science 360:eaat4422. doi: 10.1126/science.aat 4422

Peng, S.-L., Chen, C.-M., Huang, C.-Y., Shih, C.-T., Huang, C.-W., Chiu, S.-C., et al. (2019). Effects of hemodynamic response function selection on rat fMRI statistical analyses. Front. Neurosci. 13:400. doi: 10.3389/fnins.2019.00400

Petersen, C. C. H. (2017). Whole-cell recording of neuronal membrane potential during behavior. Neuron 95, 1266-1281. doi: 10.1016/j.neuron.2017.06.049

Power, R. M., and Huisken, J. (2017). A guide to light-sheet fluorescence microscopy for multiscale imaging. Nat. Methods 14, 360-373. doi: 10.1038/ nmeth.4224

Pulgar, V. M. (2018). Transcytosis to cross the blood brain barrier, new advancements and challenges. Front. Neurosci. 12:1019. doi: 10.3389/fnins. 2018.01019

Qian, M., Du, Y., Wang, S., Li, C., Jiang, H., Shi, W., et al. (2018). Highly crystalline multicolor carbon nanodots for dual-modal imaging-guided photothermal therapy of glioma. ACS Appl. Mater. Interfaces 10, 4031-4040. doi: 10.1021/ acsami.7b19716

Qian, Y., Piatkevich, K. D., Mc Larney, B., Abdelfattah, A. S., Mehta, S., Murdock, M. H., et al. (2019). A genetically encoded near-infrared fluorescent calcium ion indicator. Nat. Methods 16, 171-174. doi: 10.1038/s41592-018-0294-6

Qiao, Y., Gumin, J., MacLellan, C. J., Gao, F., Bouchard, R., Lang, F. F., et al. (2018). Magnetic resonance and photoacoustic imaging of brain tumor mediated by mesenchymal stem cell labeled with multifunctional nanoparticle introduced via carotid artery injection. Nanotechnology 29:165101. doi: 10.1088/13616528/aaaf16
Rabut, C., Yoo, S., Hurt, R. C., Jin, Z., Li, H., Guo, H., et al. (2020). Ultrasound technologies for imaging and modulating neural activity. Neuron 108, 93-110. doi: 10.1016/j.neuron.2020.09.003

Ragan, T., Kadiri, L. R., Venkataraju, K. U., Bahlmann, K., Sutin, J., Taranda, J., et al. (2012). Serial two-photon tomography for automated ex vivo mouse brain imaging. Nat. Methods 9, 255-258. doi: 10.1038/nmeth.1854

Rao, B., Zhang, R., Li, L., Shao, J.-Y., and Wang, L. V. (2017). Photoacoustic imaging of voltage responses beyond the optical diffusion limit. Sci. Rep. 7, 1-10. doi: 10.1038/s41598-017-02458-w

Raposo, G., and Marks, M. S. (2007). Melanosomes-dark organelles enlighten endosomal membrane transport. Nat. Rev. Mol. Cell Biol. 8, 786-797. doi: $10.1038 / \mathrm{nrm} 2258$

Ray, A., Wang, X., Lee, Y.-E. K., Hah, H. J., Kim, G., Chen, T., et al. (2011). Targeted blue nanoparticles as photoacoustic contrast agent for brain tumor delineation. Nano Res. 4, 1163-1173. doi: 10.1007/s12274-011-0166-1

Razansky, D., Buehler, A., and Ntziachristos, V. (2011). Volumetric real-time multispectral optoacoustic tomography of biomarkers. Nat. Protoc. 6, 11211129. doi: 10.1038/nprot.2011.351

Razansky, D., Distel, M., Vinegoni, C., Ma, R., Perrimon, N., Köster, R. W., et al. (2009). Multispectral opto-acoustic tomography of deep-seated fluorescent proteins in vivo. Nat. Photonics 3, 412-417. doi: 10.1038/nphoton.2009.98

Reiter, A., and Lediju Bell, M. A. (2017). "A machine learning approach to identifying point source locations in photoacoustic data," in Proceedings of the Photons Plus Ultrasound: Imaging and Sensing 2017 (International Society for Optics and Photonics), San Francisco, CA. doi: 10.1117/12.2255098

Renier, N., Wu, Z., Simon, D. J., Yang, J., Ariel, P., and Tessier-Lavigne, M. (2014). iDISCO: a simple, rapid method to immunolabel large tissue samples for volume imaging. Cell 159, 896-910. doi: 10.1016/j.cell.2014.10.010

Roberts, S., Seeger, M., Jiang, Y., Mishra, A., Sigmund, F., Stelzl, A., et al. (2018). Calcium sensor for photoacoustic imaging. J. Am. Chem. Soc. 140, 2718-2721. doi: 10.1021/jacs.7b03064

Ross, W. N., Salzberg, B. M., Cohen, L. B., Grinvald, A., Davila, H. V., Waggoner, A. S., et al. (1977). Changes in absorption, fluorescence, dichroism, and Birefringence in stained giant axons: : optical measurement of membrane potential. J. Membr. Biol. 33, 141-183. doi: 10.1007/BF01869514

Saleeba, C., Dempsey, B., Le, S., Goodchild, A., and McMullan, S. (2019). A student's guide to neural circuit tracing. Front. Neurosci. 13:897. doi: 10.3389/ fnins.2019.00897

Scarapicchia, V., Brown, C., Mayo, C., and Gawryluk, J. R. (2017). Functional magnetic resonance imaging and functional near-infrared spectroscopy: insights from combined recording studies. Front. Hum. Neurosci. 11:419. doi: 10.3389/fnhum.2017.00419

Schiller, J., Helmchen, F., and Sakmann, B. (1995). Spatial profile of dendritic calcium transients evoked by action potentials in rat neocortical pyramidal neurones. J. Physiol. 487(Pt 3), 583-600. doi: 10.1113/jphysiol.1995.sp0 20902

Schwalm, M., Schmid, F., Wachsmuth, L., Backhaus, H., Kronfeld, A., Aedo Jury, F., et al. (2017). Cortex-wide BOLD fMRI activity reflects locally-recorded slow oscillation-associated calcium waves. eLife 6:e27602. doi: 10.7554/eLife.27602. 030

Seeger, M., Soliman, D., Aguirre, J., Diot, G., Wierzbowski, J., and Ntziachristos, V. (2020). Pushing the boundaries of optoacoustic microscopy by total impulse response characterization. Nat. Commun. 11:2910. doi: 10.1038/s41467-02016565-2

Sepúlveda, M. R., Dresselaers, T., Vangheluwe, P., Everaerts, W., Himmelreich, U., Mata, A. M., et al. (2012). Evaluation of manganese uptake and toxicity in mouse brain during continuous $\mathrm{MnCl} 2$ administration using osmotic pumps. Contrast Media Mol. Imaging 7, 426-434. doi: 10.1002/cmmi.1469

Shang, W., Zeng, C., Du, Y., Hui, H., Liang, X., Chi, C., et al. (2017). Core-shell gold nanorod@metal-organic framework nanoprobes for multimodality diagnosis of glioma. Adv. Mater. 29:1604381. doi: 10.1002/adma.201604381

Sharon, D., and Grinvald, A. (2002). Dynamics and constancy in cortical spatiotemporal patterns of orientation processing. Science 295, 512-515. doi: $10.1126 /$ science. 1065916

Shemetov, A. A., Monakhov, M. V., Zhang, Q., Canton-Josh, J. E., Kumar, M., Chen, M., et al. (2020). A near-infrared genetically encoded calcium indicator for in vivo imaging. Nat. Biotechnol. [Epub ahead of print]. doi: 10.1038/s41587020-0710-1 
Shulman, R. G., Hyder, F., and Rothman, D. L. (2001). Cerebral energetics and the glycogen shunt: Neurochemical basis of functional imaging. Proc. Natl. Acad. Sci. U.S.A. 98, 6417-6422. doi: 10.1073/pnas. 101129298

Sieu, L.-A., Bergel, A., Tiran, E., Deffieux, T., Pernot, M., Gennisson, J.-L., et al. (2015). EEG and functional ultrasound imaging in mobile rats. Nat. Methods 12, 831-834. doi: 10.1038/nmeth.3506

Sigmund, F., Massner, C., Erdmann, P., Stelzl, A., Rolbieski, H., Desai, M., et al. (2018). Bacterial encapsulins as orthogonal compartments for mammalian cell engineering. Nat. Commun. 9:1990. doi: 10.1038/s41467-018-04227-3

Soliman, D., Tserevelakis, G. J., Omar, M., and Ntziachristos, V. (2015). Combining microscopy with mesoscopy using optical and optoacoustic label-free modes. Sci. Rep. 5:12902. doi: 10.1038/srep12902

Song, W., Xu, Q., Zhang, Y., Zhan, Y., Zheng, W., and Song, L. (2016). Fully integrated reflection-mode photoacoustic, two-photon, and second harmonic generation microscopy in vivo. Sci. Rep. 6:32240. doi: 10.1038/srep32240

Sporns, O. (2013). The human connectome: origins and challenges. Neuroimage 80, 53-61. doi: 10.1016/j.neuroimage.2013.03.023

Sporns, O., Tononi, G., and Kötter, R. (2005). The human connectome: a structural description of the human brain. PLoS Comput. Biol. 1:e42. doi: 10.1371/journal. pcbi.0010042

Staley, J., Grogan, P., Samadi, A. K., Cui, H., Cohen, M. S., and Yang, X. (2010). Growth of melanoma brain tumors monitored by photoacoustic microscopy. J. Biomed. Opt. 15:040510. doi: 10.1117/1.3478309

Steinberg, I., Huland, D. M., Vermesh, O., Frostig, H. E., Tummers, W. S., and Gambhir, S. S. (2019). Photoacoustic clinical imaging. Photoacoustics 14, 77-98. doi: 10.1016/j.pacs.2019.05.001

Stiel, A. C., Deán-Ben, X. L., Jiang, Y., Ntziachristos, V., Razansky, D., and Westmeyer, G. G. (2015). High-contrast imaging of reversibly switchable fluorescent proteins via temporally unmixed multispectral optoacoustic tomography. Opt. Lett. OL 40, 367-370. doi: 10.1364/OL.40.000367

Stosiek, C., Garaschuk, O., Holthoff, K., and Konnerth, A. (2003). In vivo twophoton calcium imaging of neuronal networks. Proc. Natl. Acad. Sci. U.S.A. 100, 7319-7324. doi: 10.1073/pnas. 1232232100

Stritzker, J., Kirscher, L., Scadeng, M., Deliolanis, N. C., Morscher, S., Symvoulidis, P., et al. (2013). Vaccinia virus-mediated melanin production allows MR and optoacoustic deep tissue imaging and laser-induced thermotherapy of cancer. Proc. Natl. Acad. Sci. U.S.A. 110, 3316-3320. doi: 10.1073/pnas.1216916110

Subach, O. M., Barykina, N. V., Anokhin, K. V., Piatkevich, K. D., and Subach, F. V. (2019). Near-infrared genetically encoded positive calcium indicator based on GAF-FP bacterial phytochrome. Int. J. Mol. Sci. 20:3488. doi: 10.3390/ ijms20143488

Sun, F., Zeng, J., Jing, M., Zhou, J., Feng, J., Owen, S. F., et al. (2018). A genetically encoded fluorescent sensor enables rapid and specific detection of dopamine in flies, fish, and mice. Cell 174, 481.e19496.e19. doi: 10.1016/j.cell.2018.06.042

Sung, Y. K., and Kim, S. W. (2019). Recent advances in the development of gene delivery systems. Biomater Res. 23:8. doi: 10.1186/s40824-019-0156-z

Tang, J., Coleman, J. E., Dai, X., and Jiang, H. (2016). Wearable 3-D photoacoustic tomography for functional brain imaging in behaving rats. Sci. Rep. 6:25470. doi: $10.1038 /$ srep 25470

Tang, J., Xi, L., Zhou, J., Huang, H., Zhang, T., Carney, P. R., et al. (2015). Noninvasive high-speed photoacoustic tomography of cerebral hemodynamics in awake-moving rats. J. Cereb. Blood Flow Metab. 35, 1224-1232. doi: 10.1038/ jcbfm.2015.138

Taruttis, A., Herzog, E., Razansky, D., and Ntziachristos, V. (2010). Real-time imaging of cardiovascular dynamics and circulating gold nanorods with multispectral optoacoustic tomography. Opt. Express 18, 19592-19602. doi: 10.1364/OE.18.019592

Tasaki, I., Watanabe, A., Sandlin, R., and Carnay, L. (1968). Changes in fluorescence, turbidity, and birefringence associated with nerve excitation. Proc. Natl. Acad. Sci. U.S.A. 61, 883-888. doi: 10.1073/pnas.61.3.883

Tong, H., Lou, K., and Wang, W. (2015). Near-infrared fluorescent probes for imaging of amyloid plaques in Alzheimer×s disease. Yao Xиe Xиe Bao 5, 25-33. doi: 10.1016/j.apsb.2014.12.006

Trigo-Mourino, P., Thestrup, T., Griesbeck, O., Griesinger, C., and Becker, S. (2019). Dynamic tuning of FRET in a green fluorescent protein biosensor. Sci. Adv. 5:eaaw4988. doi: 10.1126/sciadv.aaw 4988
Tsien, R. Y. (1980). New calcium indicators and buffers with high selectivity against magnesium and protons: design, synthesis, and properties of prototype structures. Biochemistry 19, 2396-2404. doi: 10.1021/bi00552a018

Tsien, R. Y. (1981). A non-disruptive technique for loading calcium buffers and indicators into cells. Nature 290, 527-528. doi: 10.1038/290527a0

Tzoumas, S., Deliolanis, N., Morscher, S., and Ntziachristos, V. (2014). Unmixing molecular agents from absorbing tissue in multispectral optoacoustic tomography. IEEE Trans. Med. Imaging 33, 48-60. doi: 10.1109/TMI.2013. 2279994

Vargas, J. E., Chicaybam, L., Stein, R. T., Tanuri, A., Delgado-Cañedo, A., and Bonamino, M. H. (2016). Retroviral vectors and transposons for stable gene therapy: advances, current challenges and perspectives. J. Transl. Med. 14:288. doi: 10.1186/s12967-016-1047-x

Voie, A. H., and Spelman, F. A. (1995). Three-dimensional reconstruction of the cochlea from two-dimensional images of optical sections. Comput. Med. Imaging Graph. 19, 377-384. doi: 10.1016/0895-6111(95)00034-8

Wada, K., Masujima, T., Yoshida, H., Murakami, T., Yata, N., and Imai, H. (1986). Application of photoacoustic microscopy to analysis of biological components in tissue sections. Chem. Pharm. Bull. 34, 1688-1693. doi: 10.1248/cpb.34. 1688

Wan, H., Yue, J., Zhu, S., Uno, T., Zhang, X., Yang, Q., et al. (2018). A bright organic NIR-II nanofluorophore for three-dimensional imaging into biological tissues. Nat. Commun. 9:1171. doi: 10.1038/s41467-018-03505-4

Wang, B., Xiang, L., Jiang, M. S., Yang, J., Zhang, Q., Carney, P. R., et al. (2012). Photoacoustic tomography system for noninvasive real-time three-dimensional imaging of epilepsy. Biomed. Opt. Express 3, 1427-1432. doi: 10.1364/BOE.3. 001427

Wang, H.-W., Chai, N., Wang, P., Hu, S., Dou, W., Umulis, D., et al. (2011). Labelfree bond-selective imaging by listening to vibrationally excited molecules. Phys. Rev. Lett. 106:238106. doi: 10.1103/PhysRevLett.106.238106

Wang, N., White, L. E., Qi, Y., Cofer, G., and Johnson, G. A. (2020). Cytoarchitecture of the mouse brain by high resolution diffusion magnetic resonance imaging. Neuroimage 216:116876. doi: 10.1016/j.neuroimage.2020. 116876

Wang, S., Sheng, Z., Yang, Z., Hu, D., Long, X., Feng, G., et al. (2019). Activatable small-molecule photoacoustic probes that cross the blood-brain barrier for visualization of copper(II) in mice with Alzheimer's disease. Angew. Chem. Int. Ed Engl. 58, 12415-12419. doi: 10.1002/anie.201904047

Wang, X., Chamberland, D. L., and Xi, G. (2008). Noninvasive reflection mode photoacoustic imaging through infant skull toward imaging of neonatal brains. J. Neurosci. Methods 168, 412-421. doi: 10.1016/j.jneumeth.2007.11.007

Wang, X., Pang, Y., Ku, G., Stoica, G., and Wang, L. V. (2003a). Three-dimensional laser-induced photoacoustic tomography of mouse brain with the skin and skull intact. Opt. Lett. OL 28, 1739-1741. doi: 10.1364/OL.28.001739

Wang, X., Pang, Y., Ku, G., Xie, X., Stoica, G., and Wang, L. V. (2003b). Noninvasive laser-induced photoacoustic tomography for structural and functional in vivo imaging of the brain. Nat. Biotechnol. 21, 803-806. doi: $10.1038 /$ nbt839

Wang, Y., and Wang, L. V. (2012). Förster resonance energy transfer photoacoustic microscopy. J. Biomed. Opt. 17:086007. doi: 10.1117/1.JBO.17.8.086007

Wang, Y., Xia, J., and Wang, L. V. (2013). Deep-tissue photoacoustic tomography of Förster resonance energy transfer. J. Biomed. Opt. 18;101316. doi: 10.1117/1. JBO.18.10.101316

Weber, J., Beard, P. C., and Bohndiek, S. E. (2016). Contrast agents for molecular photoacoustic imaging. Nat. Methods 13,639-650. doi: 10.1038/nmeth.3929

Wong, T. T. W., Zhang, R., Zhang, C., Hsu, H.-C., Maslov, K. I., Wang, L., et al. (2017). Label-free automated three-dimensional imaging of whole organs by microtomy-assisted photoacoustic microscopy. Nat. Commun. 8:1386. doi: 10.1038/s41467-017-01649-3

Wu, M., Chen, W., Chen, Y., Zhang, H., Liu, C., Deng, Z., et al. (2018). Focused ultrasound-augmented delivery of biodegradable multifunctional nanoplatforms for imaging-guided brain tumor treatment. Adv. Sci. 5:1700474. doi: $10.1002 /$ advs.201700474

Wu, W., Wang, P., Cheng, J.-X., and Xu, X.-M. (2014). Assessment of white matter loss using bond-selective photoacoustic imaging in a rat model of contusive spinal cord injury. J. Neurotrauma 31, 1998-2002. doi: 10.1089/neu.2014.3349

Xiang, L., Wang, B., Ji, L., and Jiang, H. (2013). 4-D photoacoustic tomography. Sci. Rep. 3:1113. doi: $10.1038 /$ srep01113 
Xu, Y., Zou, P., and Cohen, A. E. (2017). Voltage imaging with genetically encoded indicators. Curr. Opin. Chem. Biol. 39, 1-10. doi: 10.1016/j.cbpa.2017.04.005

Yagihashi, S. (2018). Clinical application of photoacoustic imaging to the evaluation of diabetic polyneuropathy. Diabetes Metab. Res. Rev. 67:582. doi: 10.2337/ db18-582-P

Yang, X., and Wang, L. V. (2008). Monkey brain cortex imaging by photoacoustic tomography. J. Biomed. Opt. 13:044009. doi: 10.1117/12. 762396

Yang, Y., Liu, N., He, Y., Liu, Y., Ge, L., Zou, L., et al. (2018). Improved calcium sensor GCaMP-X overcomes the calcium channel perturbations induced by the calmodulin in GCaMP. Nat. Commun. 9:1504. doi: 10.1038/s41467-01803719-6

Yao, D.-K., Maslov, K., Shung, K. K., Zhou, Q., and Wang, L. V. (2010). In vivo label-free photoacoustic microscopy of cell nuclei by excitation of DNA and RNA. Opt. Lett. 35, 4139-4141. doi: 10.1364/OL.35. 004139

Yao, J., Kaberniuk, A. A., Li, L., Shcherbakova, D. M., Zhang, R., Wang, L., et al. (2016). Multiscale photoacoustic tomography using reversibly switchable bacterial phytochrome as a near-infrared photochromic probe. Nat. Methods 13, 67-73. doi: 10.1038/nmeth.3656

Yao, J., Wang, L., Yang, J.-M., Maslov, K. I., Wong, T. T. W., Li, L., et al. (2015). High-speed label-free functional photoacoustic microscopy of mouse brain in action. Nat. Methods 12, 407-410. doi: 10.1038/nmeth.3336

Yao, J., and Wang, L. V. (2013). "Multi-scale multi-contrast photoacoustic microscopy," in Frontiers in Optics 2013, eds I. Kang, D. Reitze, N. Alic, and D. Hagan (Washington, DC: Optical Society of America). doi: 10.1364/FIO.2013. FM4A.1

Yao, J., and Wang, L. V. (2014). Sensitivity of photoacoustic microscopy. Photoacoustics 2, 87-101. doi: 10.1016/j.pacs.2014.04.002

Yao, J., Xia, J., Maslov, K. I., Nasiriavanaki, M., Tsytsarev, V., Demchenko, A. V., et al. (2013). Noninvasive photoacoustic computed tomography of mouse brain metabolism in vivo. Neuroimage 64, 257-266. doi: 10.1016/j.neuroimage.2012. 08.054

Yin, W., Brittain, D., Borseth, J., Scott, M. E., Williams, D., Perkins, J., et al. (2020). A petascale automated imaging pipeline for mapping neuronal circuits with high-throughput transmission electron microscopy. Nat. Commun. 11:4949. doi: 10.1038/s41467-020-18659-3

Yu, X., Qian, C., Chen, D.-Y., Dodd, S. J., and Koretsky, A. P. (2014). Deciphering laminar-specific neural inputs with line-scanning fMRI. Nat. Methods 11, 5558. doi: $10.1038 /$ nmeth. 2730

Zhang, C., Zhang, Y. S., Yao, D.-K., Xia, Y., and Wang, L. V. (2013). Labelfree photoacoustic microscopy of cytochromes. J. Biomed. Opt. 18:20504. doi: 10.1117/1.JBO.18.2.020504

Zhang, H., Wang, T., Liu, H., Ren, F., Qiu, W., Sun, Q., et al. (2019). Second nearinfrared photodynamic therapy and chemotherapy of orthotopic malignant glioblastoma with ultra-small Cu2-xSe nanoparticles. Nanoscale 11, 7600-7608. doi: 10.1039/C9NR01789E

Zhang, H. F., Maslov, K., Stoica, G., and Wang, L. V. (2006). Functional photoacoustic microscopy for high-resolution and noninvasive in vivo imaging. Nat. Biotechnol. 24, 848-851. doi: 10.1038/nbt1220

Zhang, H. K., Yan, P., Kang, J., Abou, D. S., Le, H. N. D., Jha, A. K., et al. (2017). Listening to membrane potential: photoacoustic voltagesensitive dye recording. J. Biomed. Opt. 22:45006. doi: 10.1117/1.JBO.22.4.04 5006

Zhang, P., Li, L., Lin, L., Hu, P., Shi, J., He, Y., et al. (2018). High-resolution deep functional imaging of the whole mouse brain by photoacoustic computed tomography in vivo. J. Biophotonics 11:24. doi: 10.1002/jbio.201700024

Zhang, P., Li, L., Lin, L., Shi, J., and Wang, L. V. (2019). In vivo superresolution photoacoustic computed tomography by localization of single dyed droplets. Light Sci. Appl. 8:36. doi: 10.1038/s41377-019-0147-9

Zhang, X. Y., Yang, Z. L., Lu, G. M., Yang, G. F., and Zhang, L. J. (2017). PET/MR imaging: new frontier in Alzheimer's disease and other dementias. Front. Mol. Neurosci. 10:343. doi: 10.3389/fnmol.2017.00343

Zheng, Z., Lauritzen, J. S., Perlman, E., Robinson, C. G., Nichols, M., Milkie, D., et al. (2018). A Complete Electron Microscopy Volume of the Brain of Adult Drosophila melanogaster. Cell 174, 730.e22-743.e22. doi: 10.1016/j.cell.2018. 06.019

Zhou, P., Zhao, H., Wang, Q., Zhou, Z., Wang, J., Deng, G., et al. (2018). Photoacoustic-enabled self-guidance in magnetic-hyperthermia Fe@Fe3 O4 nanoparticles for theranostics in vivo. Adv. Healthc. Mater. 7:e1701201. doi: 10.1002/adhm.201701201

Zhu, M., Sheng, Z., Jia, Y., Hu, D., Liu, X., Xia, X., et al. (2017). Indocyanine greenholo-transferrin nanoassemblies for tumor-targeted dual-modal imaging and photothermal therapy of glioma. ACS Appl. Mater. Interfaces 9, 39249-39258. doi: $10.1021 /$ acsami.7b14076

Zhu, W., Liu, K., Sun, X., Wang, X., Li, Y., Cheng, L., et al. (2015). Mn2+-doped prussian blue nanocubes for bimodal imaging and photothermal therapy with enhanced performance. ACS Appl. Mater. Interfaces 7, 11575-11582. doi: 10. 1021/acsami.5b02510

Conflict of Interest: The authors declare that the research was conducted in the absence of any commercial or financial relationships that could be construed as a potential conflict of interest.

Copyright $\odot 2021$ Bodea and Westmeyer. This is an open-access article distributed under the terms of the Creative Commons Attribution License (CC BY). The use, distribution or reproduction in other forums is permitted, provided the original author(s) and the copyright owner(s) are credited and that the original publication in this journal is cited, in accordance with accepted academic practice. No use, distribution or reproduction is permitted which does not comply with these terms. 